\title{
Connection between galactic downsizing and the most fundamental galactic scaling relations
}

\author{
E. Spitoni ${ }^{1}$, F. Calura ${ }^{2}$, M. Mignoli ${ }^{2}$, R. Gilli ${ }^{2}$, V. Silva Aguirre ${ }^{1}$, and A. Gallazzi ${ }^{3}$ \\ 1 Stellar Astrophysics Centre, Department of Physics and Astronomy, Aarhus University, Ny Munkegade 120, 8000 Aarhus C, \\ Denmark \\ e-mail: spitoni@phys.au.dk \\ 2 INAF-OAS, Osservatorio di Astrofisica e Scienza dello Spazio di Bologna, via Gobetti 93/3, 40129 Bologna, Italy \\ 3 INAF - Osservatorio Astrofisico di Arcetri, Largo Enrico Fermi 5, 50125 Firenze, Italy
}

Received 4 March 2020 / Accepted 31 July 2020

\begin{abstract}
Context. In their evolution, star-forming galaxies are known to follow scaling relations between some fundamental physical quantities, such as the relation between mass metallicity and star formation main sequence.

Aims. We study the evolution of galaxies that at a given redshift, lie simultaneously on the mass-metallicity and main-sequence relations (MZR, MSR).

Methods. To this aim, we used the analytical leaky-box chemical evolution model, in which galaxy evolution is described by the infall timescale $\tau$ and the wind efficiency $\lambda$. We provide a detailed analysis of the temporal evolution of their metallicity, stellar mass, mass-weighted age, and gas fraction.

Results. The evolution of the galaxies lying on the MZR and MSR at $z \sim 0.1$ suggests that the average infall timescale in two different bins of stellar masses $\left(M_{\star}<10^{10} M_{\odot}\right.$ and $\left.M_{\star}>10^{10} M_{\odot}\right)$ decreases with decreasing redshift through the addition of new galaxies with shorter timescales. This means that at each redshift, only the youngest galaxies can be assembled on the shortest timescales and still belong to the star-forming MSR. In the lowest mass bin, a decrease in median $\tau$ is accompanied by an increase in the median $\lambda$ value. This implies that systems that formed at more recent times will need to eject a larger amount of mass to retain their low metallicity values. Another important result is that galactic downsizing, as traced by the age-mass relation, is naturally recovered by imposing the local MZR and MSR for star-forming galaxies. This result is retained even when a constant star formation efficiency for different galactic masses is assumed (without imposing the observed scaling relation between stellar mass and gas-depletion timescales). Finally, we study the evolution of the hosts of C IV-selected active galactic nuclei, which at $z \sim 2$ follow a flat MZR. When we impose that these systems lie on the MSR, we find an "inverted" MZR at lower redshifts, meaning that some additional processes must be at play in their evolution.

Conclusions. In our model, galactic downsizing is a direct consequence of the MZR and MSR for star-forming galaxies. This poses a challenge for models of galaxy evolution within a cosmological framework.
\end{abstract}

Key words. galaxies: abundances - galaxies: evolution - galaxies: fundamental parameters - ISM: abundances

\section{Introduction}

In the past decades, several observational campaigns have confirmed that the evolution of star-forming galaxies is described by scaling relations between some fundamental physical quantities. For instance, a tight relation has been found between galactic stellar mass and metallicity (Lequeux et al. 1979; Maiolino \& Mannucci 2019), the so-called mass-metallicity relation (hereinafter MZR). In the local Universe, the assessment of the MZR and other scaling relations was possible through the high-quality data collected within the Sloan Digital Sky Survey (SDSS), which have enabled us to measure the flux ratios of the main optical emission lines for more than 100000 galaxies (e.g., Tremonti et al. 2004; Mannucci et al. 2010; Pérez-Montero et al. 2013; Lian et al. 2015).

Moreover, the observed evolution with redshift of the MZR (i.e., Maiolino et al. 2008; Yuan et al. 2013; Zahid et al. 2014) allows us to trace the chemical enrichment history of galaxies throughout different cosmic epochs. Zahid et al. (2014) pointed out that at redshifts $z<1.6$, the MZR follows a steep slope with a knee at a characteristic turnover mass at $M_{*} \sim 10^{10} M_{\odot}$. At stellar mass values higher than this, the MZR flattens because in the most massive galaxies, the metallicities begin to saturate. As a consequence, the redshift evolution of the MZR depends only on the evolution of the characteristic turnover mass. The evolving turnover mass can be related to the change in the ratio of gas to stellar mass (see, e.g. also the similar dependence between turnover mass and star formation rate, SFR, in Curti et al. 2020). Different theoretical scenarios have been presented in the past in order to explain the MZR, and they are summarized below.

(i) Galactic outflows: Low-mass galaxies are more efficient in expelling metal-enriched matter than giant galaxies because the gravitational potential wells of the former are shallower (Larson 1974; Tremonti et al. 2004; Spitoni et al. 2010, 2017b; Hirschmann et al. 2016). In the framework of pure chemical evolution models, Lian et al. (2018b) have suggested that strong metal outflows that occur in the earliest galactic evolutionary phases also help reconciling gas phase and mass-weighted stellar MZRs.

(ii) Variable star formation efficiency (SFE): The efficiency of star formation is higher in more massive systems, which have formed the bulk of their stars by means of an intense star 
formation event at high redshift, quickly enriching their interstellar medium (ISM) to solar or supersolar metallicities.

In the local Universe, Boselli et al. (2014) presented a scaling relation between the typical galaxy gas-depletion timescale and the galaxy stellar mass that can be easily transformed into SFE. The authors observed that massive galaxies consume the available gas reservoir on shorter typical timescales than dwarf galaxies. This means that larger galaxies are expected to experience higher SFEs on average (see also Matteucci 2012). Various chemical evolution studies support this scenario (Matteucci 1994; Lequeux et al. 1979; Calura et al. 2009).

(iii) A third interpretation of the MZR relation is linked to the initial stellar mass function. Köppen et al. (2007) showed how the MZR relation can be explained by a higher upper mass cutoff in the initial mass function (IMF) in more massive galaxies.

Chemical evolution models are powerful tools that help to probe fundamental processes that regulate galaxy formation and evolution. They can provide important constraints on how subsequent stellar generations have modified the chemical composition of the ISM to give place to chemical abundance pattern as observed in present-day galaxies (Matteucci 2012). In this framework, Spitoni et al. (2017b) presented an analytical solution for the evolution of the galaxy metallicity, where an exponential infall of gas was assumed for the gas accretion rate, along with instantaneous mixing and instantaneous recycling approximation (IRA; Matteucci 2012).

In their study of the MZR in the local SDSS star-forming galaxies by Peng et al. (2015), Spitoni et al. (2017b) found that lower mass galaxies suffered more intense winds than higher mass galaxies, in agreement with scenario (i) mentioned above. They also had to be characterized by shorter timescales of gas accretion, imposing the variable SFE by Boselli et al. (2014), as indicated by scenario (ii).

Another fundamental quantity to understand galaxy evolution is the SFR, which helps to shed light on the basic processes regulating the conversion of gas into stars and the growth of the stellar mass. Observations of large samples of starforming galaxies in different redshift intervals have allowed us to establish the existence of a well-defined relation between the SFR and the stellar mass, the so-called main-sequence relation (MSR), which has been thoroughly studied in several works (Brinchmann et al. 2004; Elbaz et al. 2007; Noeske et al. 2007; Peng et al. 2010; Santini et al. 2017; Pearson et al. 2018). Like the MZR, the MSR is also characterized by a clear evolution with redshift. Pearson et al. (2018) studied the MSR in the redshift range between $z=0.2-6$, showing that the slope of this relation does not change substantially with cosmic time. In particular, the slope of the MSR only becomes steeper for galaxies at high redshift, in the range between $3.8 \leq z \leq 6$. On the other hand, it has been ascertained in several studies that the zero-point of the MSR increases with redshift (e.g., Santini et al. 2017; Iyer et al. 2018).

One of the most remarkable results of studies of scaling relations in local and distant galaxies was the discovery of substantial differences in the star formation history (SFH) of low- and high-mass galaxies, also known as galaxy downsizing (DS; e.g., Cowie et al. 1996; Mortlock et al. 2011). In the framework of galactic DS, the largest galaxies are assembled at the earliest times, with high SFR values mostly concentrated in their earliest phases and accompanied by very efficient chemical enrichment (Calura et al. 2009; Matteucci 2012; Maiolino \& Mannucci 2019). The phenomenology of galactic downsizing is wide and multifaceted, as is reflected by several observational aspects of galaxies at both low and high redshift, including color distribu- tions at optical and infrared wavelengths of high-redshift galaxies (Cowie et al. 1996), studies of the integrated abundance ratios in local earl- type galaxies (e.g., Spolaor et al. 2010 and references therein), studies of the evolution of the galactic stellar mass function (e.g., Mortlock et al. 2011), and studies of metallicity, age, and stellar mass from optical spectra of local galaxies (Gallazzi et al. 2005) All together, these independent pieces provide the same indications: a more intense star formation activity in the most massive galaxies at early epochs, followed by higher star formation in low-mass galaxies at lower redshifts.

By means of the analytic chemical evolution model of Spitoni et al. (2017b), our aim is to study the evolution of some basic properties of the galaxies that build the MZR and MSR at different redshifts. These properties include stellar mass and star formation activity, and how these are related to other quantities, such as the ages of the stellar populations. In performing this analysis, another aspect to be investigated is the connection between the fundamental scaling relations mentioned above (the MZR and MSR) and galactic DS.

We also show the backward temporal evolution of the principal physical properties (i.e., gas fraction, metallicity, SFR, and stellar mass) of star-forming galaxies that are part of the local MZR and MSR. Other studies investigated the backward evolution of the MZR in star-forming galaxies with chemical evolution models (Calura et al. 2009; Lian et al. 2018a), but without focus on the interplay with other scaling relations and on the particular role played by the MS.

We also analyze the temporal evolution and the fate of the galaxies with stellar masses, metallicities, and SFRs constrained by the high-redshift MZRs and MSRs. In particular, we discuss the implications of the newly observed flat MZR at redshift $z=$ 2.2 by Mignoli et al. (2019), who analyzed the metal content of $88 \mathrm{C}$ IV-selected galaxies containing type 2 AGN with reliable measurements at high redshift.

Our paper is organized as follows. In Sect. 2 we recall the main features of the chemical evolution model of Spitoni et al. (2017b). In Sect. 3 we describe the method we used to create our grid of models. In Sect. 4 we present the main observational constraints and in Sect. 5 our main results assuming a Salpeter (1955) initial mass function (IMF). Our conclusions are drawn in Sect. 6.

\section{Model by Spitoni et al. (2017b)}

Spitoni et al. (2017b) presented new analytical solutions in presence of an infall of gas that follows an exponential law for the metal abundances, gas, and stellar masses within a galactic evolutionary framework where instantaneous mixing and IRA are assumed (Matteucci 2012). The infall of gas that follows an exponential law is a fundamental assumption that is adopted in most of the numerical chemical evolution models in which IRA is relaxed. Chemical evolution models of our Galaxy (Calura et al. 2009; Romano et al. 2010; Grisoni et al. 2018; Vincenzo et al. 2019; Spitoni et al. 2016, 2017a, 2019a,b, 2020) assume that the various different stellar components formed by means of separate accretion episodes of gas, with the accretion rate of each episode expressed by an exponential law.

Spitoni et al. (2017b) modeled the SFR by means of the Schmidt (1959) law, where the star formation rate SFR $\psi(t)$ can be expressed as $\psi(t)=S \cdot M_{\text {gas }}(t)$, where $M_{\text {gas }}(t)$ is the gas mass at the time $t$ and $S$ is the so-called SFE, which is commonly expressed in $\mathrm{Gyr}^{-1}$ and which is a free parameter of the model. 
The gas infall rate is expressed by an exponential law $\mathcal{I}(t)=$ $A e^{-t / \tau}$, where $\tau$ is the infall timescale. The quantity $A$ is a constant, constrained by the total infall gas mass $M_{\text {inf }}$ (Spitoni et al. 2017b)

In the model proposed by Spitoni et al. (2017b), gas outflows in galaxies have been taken into account as well. The outflow rate is proportional to the SFR in the galaxy (see Recchi et al. 2008; Spitoni et al. 2010; Spitoni 2015): $W(t)=\lambda \cdot \psi(t)$, with $\lambda$ being the loading factor parameter (a dimensionless quantity).

The analytical solution of Spitoni et al. (2017b) for the evolution of the gas-phase metallicity, defined as $Z=M_{Z} / M_{\mathrm{gas}}$, is

$$
\begin{aligned}
Z(t)= & \frac{y_{z} S(1-R)}{\alpha \tau-1} . \\
& \frac{M_{\mathrm{gas}}(0) t(\alpha \tau-1)^{2}+A \tau\left[t-\tau(1+\alpha t)+\tau \mathrm{e}^{\alpha t-t / \tau}\right]}{A \tau\left(\mathrm{e}^{\alpha t-t / \tau}-1\right)+M_{\mathrm{gas}}(0)(\alpha \tau-1)},
\end{aligned}
$$

in which it is assumed that the infalling gas is metal free, as is the galaxy at the epoch of its formation. In Eq. (1) we introduce the parameter $\alpha$, defined as $\alpha \equiv(1+\lambda-R) S$. The quantities $y_{Z}$ and $R$ are the so-called yield per stellar generation and returned mass fraction (for a detailed description, see Spitoni et al. 2017b), respectively.

The values of $y_{Z}$ and $R$ are average values computed at various metallicities and for various IMFs, and are from Table 2 of Vincenzo et al. (2016), in which the compilation of stellar yields of Romano et al. (2010) was considered. As for the stellar IMF, two different cases are studied in this work. In the case of a Salpeter (1955) IMF, we assume $R=0.287$ and $y_{\mathrm{O}}=0.018$, whereas for a Chabrier (2003) IMF (see Appendix A), $R=0.441$ and $y_{\mathrm{O}}=0.0407$, where $y_{\mathrm{O}}$ is the oxygen yield per stellar generation. The other analytical expressions for the evolution of the total mass, gas mass and stellar mass can be found in Spitoni et al. (2017b).

More recently, Weinberg (2017) presented chemical analytical solutions adopting a different approach than Spitoni et al. (2017b). They specified the SFR history instead of an analytical form for the infalling gas. The SFR derived by an exponential infalling gas rate in Spitoni et al. (2017b) is similar to the linearexponential SFR history (proportional to $t \mathrm{e}^{-t / \tau_{\text {sh }}}$, where $\tau_{\text {sfh }}$ is the timescale of the exponential decline) presented by Weinberg (2017), and the results for the metallicity evolution are qualitatively similar.

\section{Method}

We focused on a given redshift, and we imposed that the galaxies must follow the observed MZR and the star-forming MSR measured at that redshift (see Sect. 4 for details concerning the observational constraints). First, we study the properties of the local galaxies by imposing the MZR and the MSR at redshift $z=0.1$, and analyzing their backward evolution toward higher redshifts. On the other hand, by imposing the same scaling relations at higher redshift, we computed a forward evolution of the galaxies at lower redshifts and towardsthe present time. In this framework, we also study the evolution of the galaxies that at redshift $z=2.2$ follow the flat MZR as derived by Mignoli et al. (2019).

A grid of galactic models was created by varying some key parameters within a wide but realistic space and with a fine resolution. The values adopted for the infall timescale $\tau$ span a range between $0.05 \mathrm{Gyr}$ and $10 \mathrm{Gyr}$, with a resolution $\Delta \tau=0.05 \mathrm{Gyr}$ as in Spitoni et al. (2017b). The wind parameter $\lambda$ was varied in the range between 0 and 10 , with a resolution of $\Delta \lambda=0.05$, which is a ten times finer resolution than was adopted in Spitoni et al. (2017b), where $\Delta \lambda=0.5$. Moreover, as in Spitoni et al. (2017b), the total infall mass in the Salpeter (1955) case varied between $10^{7.5}$ and $10^{11.5} M_{\odot}$. In the case of the Salpeter (1955) IMF, we varied the infall masses according to the following expression:

$M_{\mathrm{inf}}=(k / 10) \cdot 10^{j+7.5} M_{\odot}$,

where $1 \leq k \leq 100$, and in steps $\Delta k=5,1 \leq j \leq 3$, and in steps $\Delta j=1$. In Appendix A we discuss the effects of a different IMF on this parameter. Each galaxy was evolved in time with a resolution of $\Delta t=0.036 \mathrm{Gyr}$.

Following Spitoni et al. (2017b), we did not assume that all galaxies are coeval, but we allowed them to form at different redshifts. At a fixed stellar mass, a galaxy was considered part of the local (or high-redshift) MZR and MSR if the differences between the predicted oxygen abundance and SFR values and the observed values were smaller than $10^{-3}$ : that is, $\Delta(\mathrm{O} / \mathrm{H})=(\mathrm{O} / \mathrm{H})_{\text {model }}-(\mathrm{O} / \mathrm{H})_{\text {data }}<10^{-3}$ dex and $\Delta \log (\mathrm{SFR})=$ $\log \left(\mathrm{SFR}_{\text {model }}\right)-\log \left(\mathrm{SFR}_{\text {data }}\right)<10^{-3} \log \left(M_{\odot} \mathrm{yr}^{-1}\right)$. In this way, we assumed zero scatter in the MZR and MSR.

Moreover, in order to be consistent with the observational data by Trussler et al. (2020), we defined the average massweighted age following Calura et al. (2014). For each galaxy, characterized at a certain evolutionary time $t$ by an SFR $\psi(t)$, we computed the average mass-weighted age at the time $t_{n}$ as

$\operatorname{Age}\left(t_{n}\right)=\frac{\int_{0}^{t<t_{n}}\left(t_{n}-t\right) \psi(t) \mathrm{d} t}{\int_{0}^{t<t_{n}} \psi(t) \mathrm{d} t}$.

Average mass-weighted ages were computed at four different redshift values, each corresponding to a different value for $t_{n}$. The SFE was constrained by means of the scaling relation for local galaxies presented by Boselli et al. (2014), which links the typical galaxy gas-depletion timescale, $\tau_{\text {gas }}$, and the galaxy stellar mass as follows:

$\log \left(\tau_{\text {gas }}\right)=-0.73 \log \left(M_{\star} / M_{\odot}\right)+16.75$,

where $\tau_{\text {gas }}=M_{\text {gas }} /$ SFR is defined as the inverse of our SFE, namely $\tau_{\text {gas }}=1 / S$. According to Eq. (4), galaxies with higher stellar mass would consume their available gas mass on shorter typical timescales if only star formation activity were taking place in the galaxy; this means that larger galaxies are expected to experience higher SFEs on average (see Matteucci 2012).

In our standard model, we adopted Eq. (4) to constrain the galaxy SFE (which was kept fixed during the galaxy evolution), given an initial value for the galaxy infall mass, $M_{\text {inf }}$ (in the right panel of Fig. 1 the variation of the SFE is shown as a function of the total gas infall mass $M_{\text {inf }}$ ). The role of this assumption is discussed in Sect. 5.1.4. We also consider another case in which a constant SFE as a function of galaxy mass is assumed.

\section{Observed scaling relations at different redshifts}

In this section we describe the observational constraints for the MZR and the star-forming MSRs at different redshifts. The observed MZR at redshift $z \sim 0.1$ is the one by Kewley \& Ellison (2008), as fit by Maiolino et al. (2008) with a calibration 

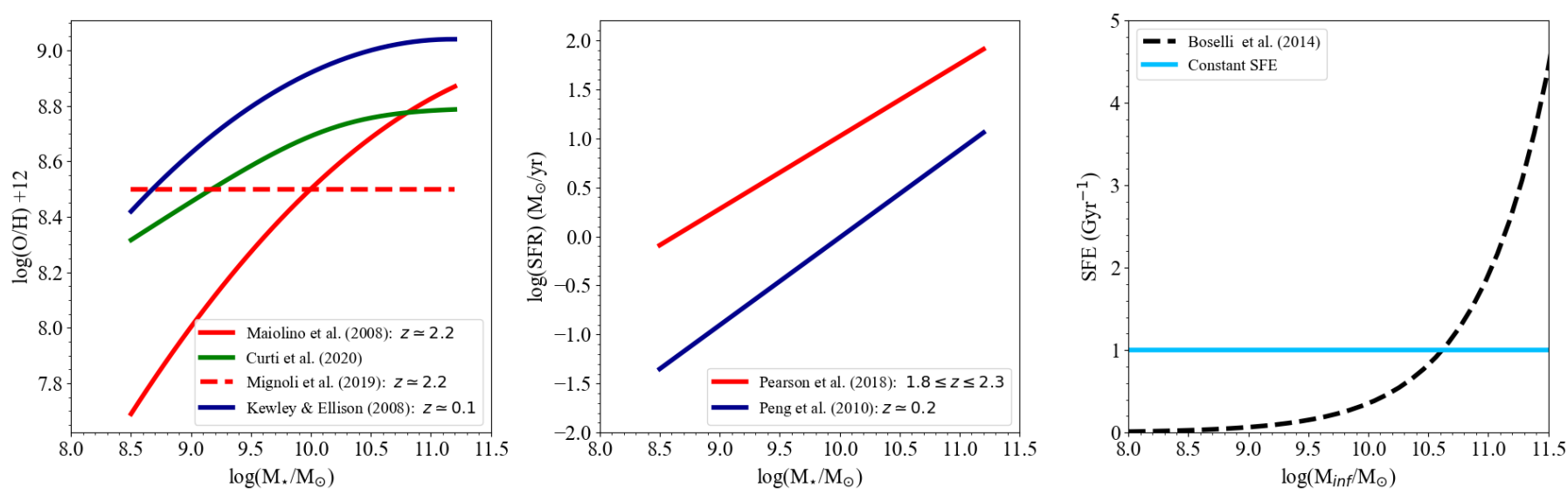

Fig. 1. Left panel: observed MZRs adopted at different redshifts: the local MZR by Kewley \& Ellison (2008) is depicted with the blue line, and the high-redshift MZR by Maiolino et al. (2008) with the red line. The dashed red line is the MZR observed by Mignoli et al. (2019) at $z \sim 2$. With the solid green line we also show the local MZR relation for SDSS galaxies found by Curti et al. (2020) with a fully $T_{\mathrm{e}}$-based abundance scale analysis. Middle panel: star-forming main sequence by Peng et al. (2010) in local SDSS galaxies shown with the blue line, and the high-redshift relation is from Pearson et al. (2018). Right panel: with the dashed black line we show the SFE as a function of the infall mass $M_{\text {inf }}$ from the scaling relation for the gas-depletion timescale as found by Boselli et al. (2014). The constant SFE fixed at the value of $1 \mathrm{Gyr}^{-1}$ is indicated with the light blue curve.

based on photoionization models provided by Kewley \& Dopita (2002):

$\log (\mathrm{O} / \mathrm{H})+12=-0.0864 \cdot\left[\log \left(\frac{M_{\star}}{M_{\odot}}\right)-\alpha\right]^{2}+\beta$,

where $\alpha$ and $\beta$ are the free parameters of the fit that were fixed at the values of $\alpha=11.18$ and $\beta=9.04$.

In Sect. 5.1.5 we show the sensitivity of our results to the adoption of a different calibration method for metallicity measures. In particular, we show the effects of adopting a $T_{\mathrm{e}}$-based metallicity calibration for the local MZR.

As for the high-redshift MZR, we considered two different relations. The first has been presented by Maiolino et al. (2008) for galaxies at redshift $z=2.2$, for which the analytical fit form is the same as Eq. (5) but with $\alpha=12.38$ and $\beta=8.99$.

Concerning high-redshift objects, Maiolino et al. (2008) underlined that at this time, no single strong-line calibration method existed over the wide metallicity range spanned by such galaxies. Therefore, at low metallicities $(12+\log (\mathrm{O} / \mathrm{H})<8.35)$, they adopted calibrations of strong-line diagnostics based on the $T_{\mathrm{e}}$ method, and at higher metallicity, they relied on photoionization models provided by Kewley \& Dopita (2002). In our analysis, we also considered the recent results by Mignoli et al. (2019), in which a flat $\mathrm{MZR}$ with $\log (\mathrm{O} / \mathrm{H})+12 \simeq 8.5$ dex was found for galaxies hosting Type $2 \mathrm{AGN}$ at $z \simeq 2.2$.

Finally, we present the scaling relations of the star-forming main sequence that we used as a further constraint of our models. At low redshift, the MSR is adopted from Peng et al. (2010), computed at redshift $z=0.2$,

$\log \left(\mathrm{SFR} / M_{\odot} \mathrm{yr}^{-1}\right)=0.89 \cdot \log \left(\frac{M_{\star}}{M_{\odot}}\right)-8.93$.

The high-redshift $(1.8 \leq z \leq 2.3)$ star-forming MSR is taken from Pearson et al. (2018), fit by the following expression:

$\log \left(\mathrm{SFR} / M_{\odot} \mathrm{yr}^{-1}\right)=0.74 \cdot\left[\log \left(\frac{M_{\star}}{M_{\odot}}\right)-10.5\right]+1.39$.

In the left and middle panels of Fig. 1 we show the observed MZRs and the MSRs, respectively.

\section{Model results}

Our aim is to reconstruct the past SFH of the galaxies that obey the two fundamental scaling relations considered here, that is, the MZR and the MSR of star-forming galaxies, at two representative different redshifts. The redshifts at which we performed our analysis were those that already include significant samples of star-forming galaxies and for which the MZR has been evaluated homogeneously, that is, using the same method for the stellar mass and the interstellar metallicity, as described in Calura et al. (2009).

The properties of the galaxies that follow the MZR and MSR at these representative redshifts were studied at different epochs: the backward evolution of the galaxies thath obey the observed MZR and MSR at $z \sim 0.1$, and the forward evolution of objects that at redshift $z \sim 2.2$ are part of both the MSR and the MZR. Our aim is to track the evolution of the galaxies that in a given phase of their history simultaneously followed the MZR and the MSR.

\subsection{Backward evolution}

\subsubsection{MZR and star-forming MSR at $z=0.1$}

Figures 2 and 3 show the backward evolution of the galaxies that obey the analytical fit of the observed MZR at $z \sim 0.1$ of Kewley \& Ellison (2008), as reported by Maiolino et al. (2008) and the main sequence of star-forming galaxies as derived by Peng et al. (2010). In Figs. 2 and 3, the galaxies are color-coded as a function of their loading factor $\lambda$ and infalling gas mass, respectively.

By imposing that they obey the Peng et al. (2010) MSR and by means of the Schmidt (1959) law, which links the gas mass to the SFR, we correctly retrieve the anticorrelation between a fundamental quantity related to the gas-accretion history of galaxies, that is, the gas fraction $\mu \equiv M_{\text {gas }} /\left(M_{\text {gas }}+M_{\star}\right)$, and the stellar mass. It is important to note that this anticorrelation is a well-established empirical relation of galaxies found at various redshifts. Locally, this anticorrelation was found by Kannappan (2004), who determined the gas fraction for 35000 galaxies from the SDSS and the Two Micron All Sky Survey 

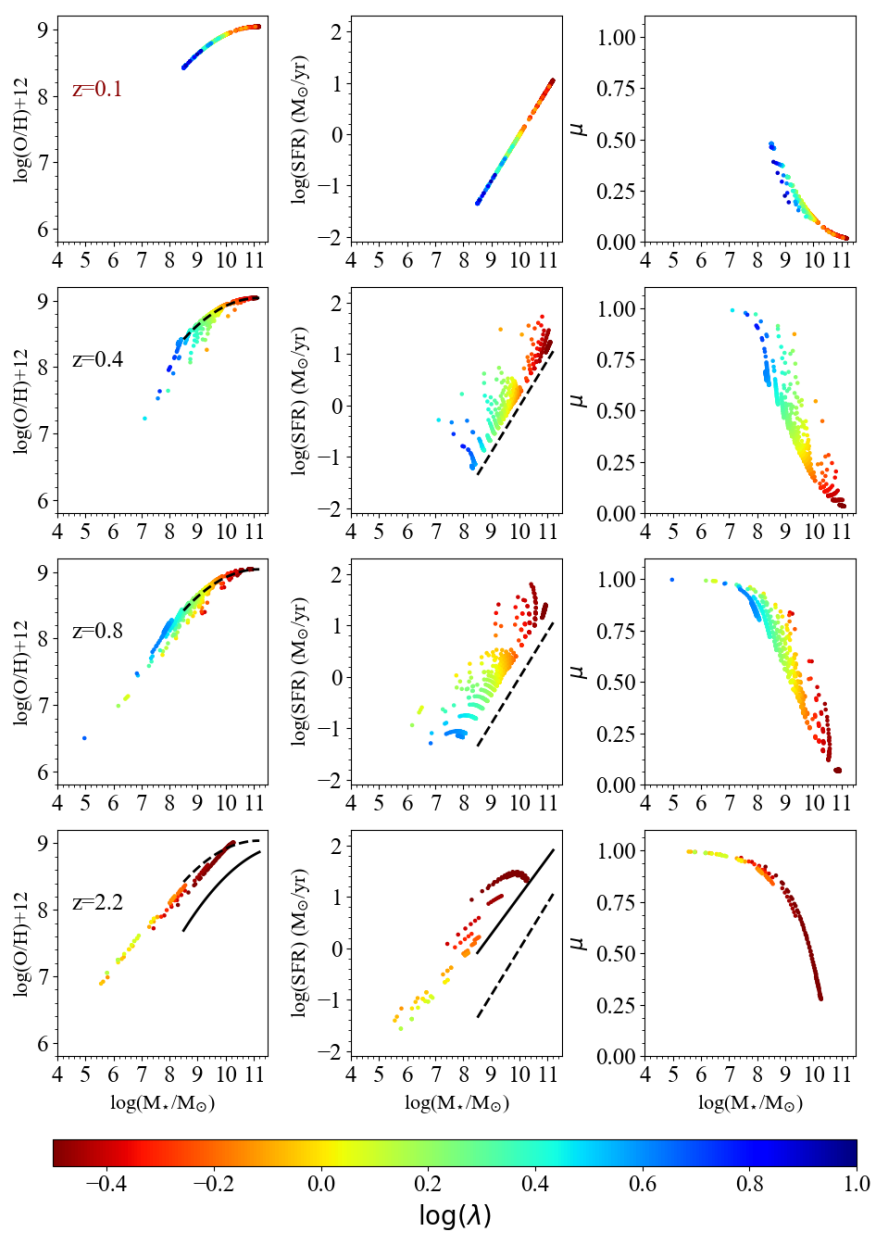

Fig. 2. Backward evolution from $z=0.1$ to $z=2.2$ of the galaxies that obey the analytical fit of the observed MZR at $z \sim 0.1$ of Kewley \& Ellison (2008), as reported by Maiolino et al. (2008) and the local main sequence of star-forming galaxies as derived by Peng et al. (2010). In the first, second, and third column we show the evolution of MZR, SFR vs. stellar mass, and gas fraction vs. stellar mass evolution, respectively. The color-coding indicates the loading factor parameter $\lambda$. The solid black lines in the bottom left and middle panels indicate the MZR and MSR observed at redshift $z=2.2$, respectively. The dashed black lines in the panels of the first column and in each SFR $\log \left(M_{\star} / M_{\odot}\right)$ plot show the MZR at redshift $z \sim 0.1$ of Kewley \& Ellison (2008) and the MSR derived in local star-forming galaxies by Peng et al. (2010), respectively.

(2MASS) databases on the basis of photometric techniques. A similar study on a smaller sample of low-mass galaxies has been performed by Geha et al. (2006) and the results of Kannappan (2004) were confirmed. The slope of the curve steepens from low-mass to high-mass systems, which is generally interpreted with decreasing gas consumption timescales from dwarf to giant galaxies (Calura et al. 2008).

The past evolution of our model galaxies was computed at three different redshifts, $z=0.4,0.8$, and 2.2. The higher redshift value in the plot is the value at which a substantial number of galaxies have appeared that are now forming stars and build the local MZR. The epoch at which each galaxy must be born is a result of our method, which stems directly from Eq. (1) and from the imposition of the observational constraints.

A significant evolution of all the three quantities shown in Fig. 2 is evident from the plot. A positive correlation between stellar mass and metallicity is already in place at $z=2.2$. In the young galaxies present at that redshift, the shape of the MZR is
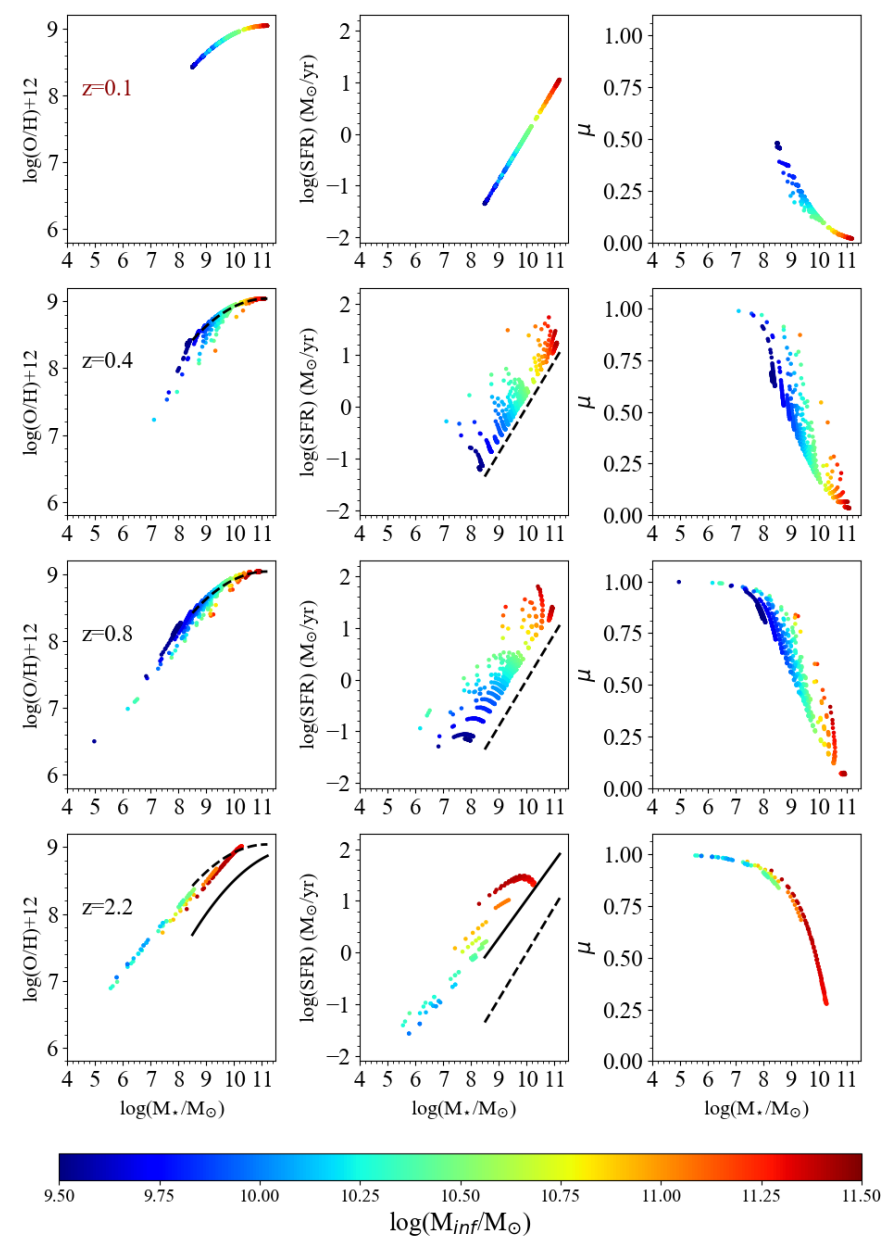

Fig. 3. As in Fig. 2, but the color-coding indicates the total infalling gas mass.

missing the characteristic plateau at high masses that is generally observed at all redshifts. This can be understood by considering that the population at $z=2.2$ as shown in Fig. 2 is only a subsample of the entire population of all the star-forming galaxies at this redshift that obey the MZR but that also include many systems that now are passive, that is, that do not belong to the local MSR relation, and that build the high-mass plateau at this redshift, as shown in Savaglio et al. (2005) and Maiolino et al. (2008).

The relation between SFR and mass appears appreciably flatter than at $z=0.1$, and even if the gas fraction still anticorrelates with stellar mass, it is characterized by an inverted curvature with respect to the local relation. All the galaxies that build the three relations are characterized by very low wind efficiencies $(\log (\lambda) \leq-0.4)$ and by the high infall mass values $\left(\log \left(M_{\mathrm{inf}} / M_{\odot}\right) \sim 11.5\right)$.

At $z=0.8$, the MZR appears to be more extended than at $z=2.2$, in particular in that it is much more populated at $\log \left(M_{\star} / M_{\odot}\right) \geq 8$. At this redshift, the hint of a plateau has already appeared in the MZR at the highest mass values, namely at $\log \left(M_{\star} / M_{\odot}\right)>10$. The appearance of the plateau may be interpreted as a flattening of the SFH of the most massive galaxies, which are characterized by the highest SFR values and shortest gas-consumption timescales (see Fig. A.6). As a consequence of a faster gas consumption in the largest galaxies, their present-day metallicity is reached at earlier times than in 

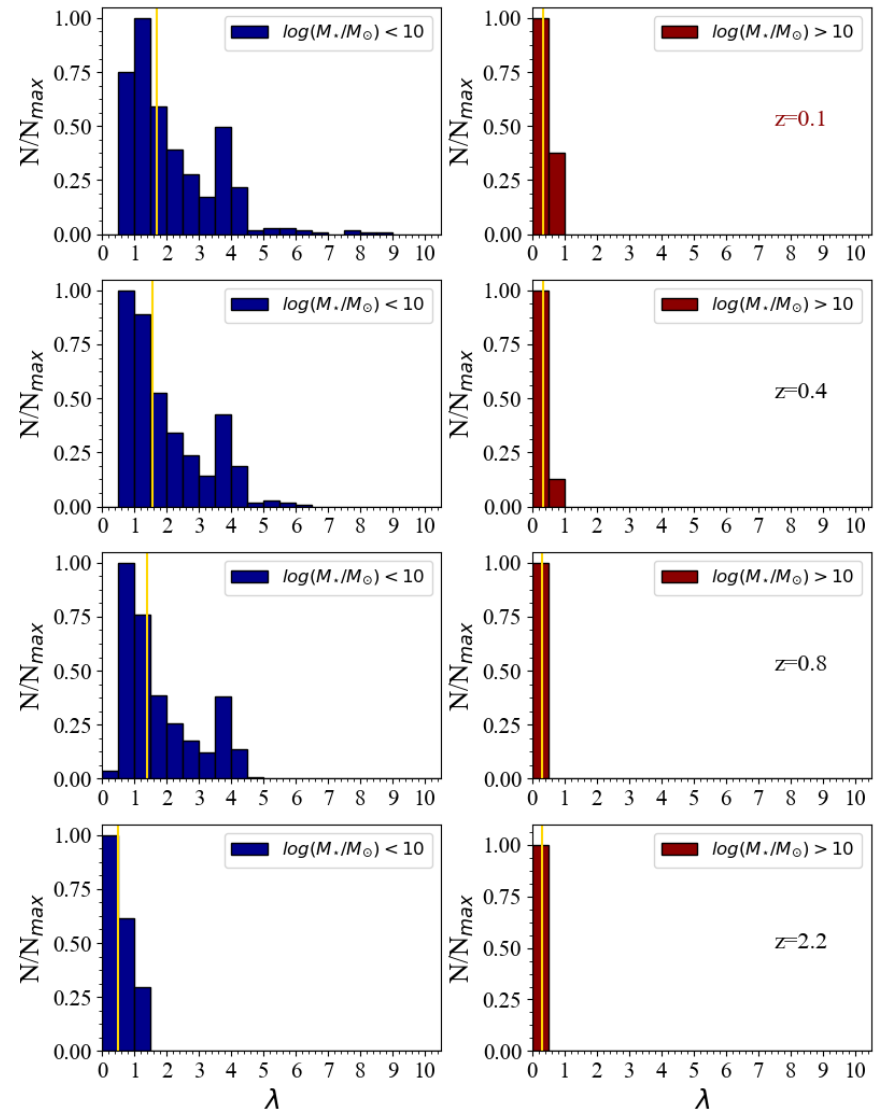

Fig. 4. Distribution of the loading factor parameter $\lambda$ for the galaxies of Fig. 2 computed at redshifts $z=2.2, z=0.8, z=0.4$, and $z=0.1$ for two different bins of stellar mass. The left panels show distributions for objects with stellar mass $\log \left(M_{\star} / M_{\odot}\right)<10$, and the right panels indicate distributions for stellar mass $\log \left(M_{\star} / M_{\odot}\right)>$ 10. The yellow vertical lines indicate the median values of each distribution

low-mass galaxies. The SFR- $M_{*}$ relation of the galaxies at $z=$ 0.8 is also more extended and more scattered than at $z=2.2$.

The $\mu-M_{\star}$ relation sees the appearance of a significant population of newly born, extremely gas-rich and metal-poor galaxies characterized by $\mu \sim 1$ at $\log \left(M_{*} / M_{\odot}\right)<8$. The range of the wind parameter and infall mass values presented by the galaxies at $z=0.8$ also have extended considerably with respect to $z=2.2$. The maximally gas-rich galaxies present the highest values of the $\lambda$ parameter $(\log \lambda \sim 0.6)$ and are also characterized by the lowest infall mass values.

On the other hand, the strong evolution experienced by the largest systems causes them to have very low gas fractions.

At $z=0.4$ the horizontal extension of the MZR has reduced, as all galaxies have grown in stellar mass. The MZR is now much more similar to the local one, with the exception of a larger scatter at low masses, which disappears later by construction. The population of galaxies with gas fractions $\sim 1$ is considerably reduced. The gas fraction-mass relation shows a parabolic behavior at the highest stellar masses, with an inflection at $\log \left(M_{*} / M_{\odot}\right) \sim 9$.

In Figs. 4 and 5 we present the distribution of the loading factor parameter $\lambda$ and the infall timescale parameter $\tau$ at different redshifts for the model galaxies of Figs. 2 and 3. The distribution are computed in two different bins of stellar masses $\left(M_{\star}<10^{10} M_{\odot}\right.$ and $\left.M_{\star}>10^{10} M_{\odot}\right)$.
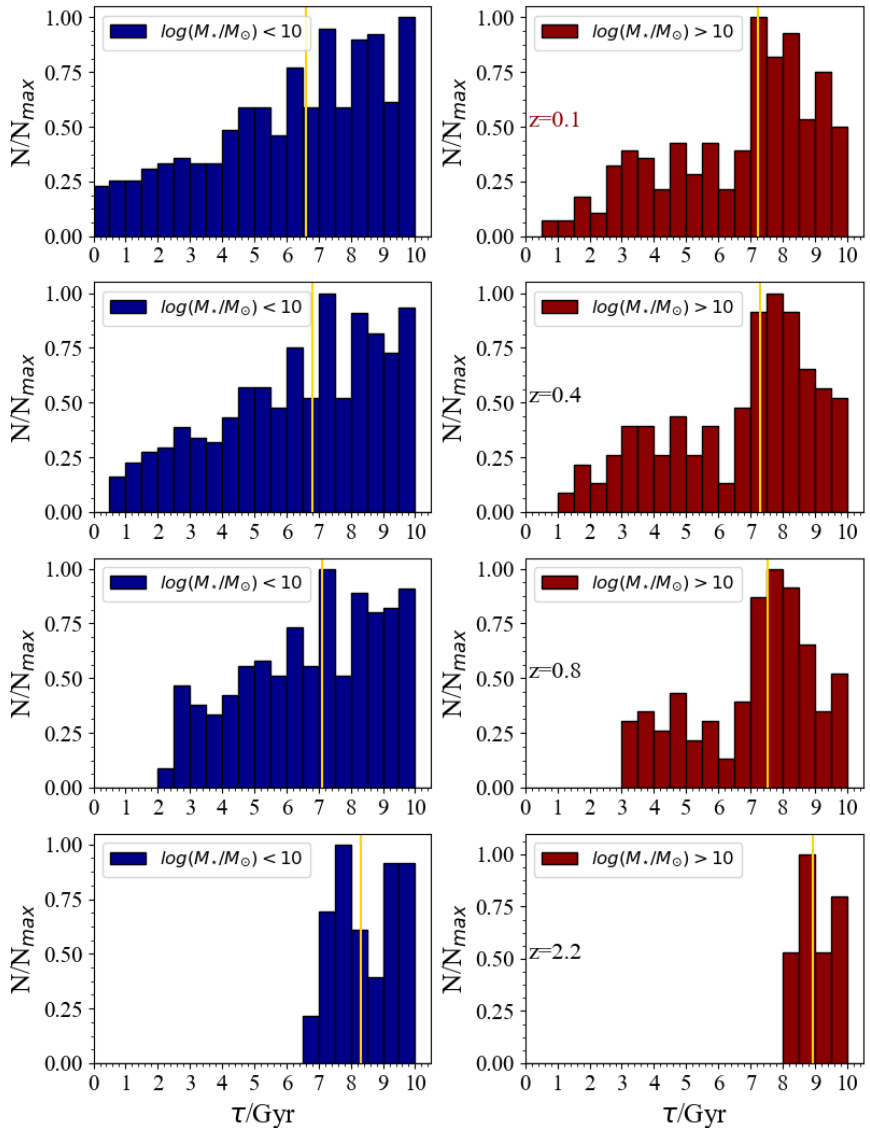

Fig. 5. As in Fig. 4, but in each panel the plotted distribution is for the infall timescale parameter $\tau$.

The galaxies present at redshift $z=2.2$ are characterized by weak winds and long timescales of gas accretion. At low redshift, these objects have to maintain a large reservoir of gas in order to be part of the local MSR.

Galaxies in the higher mass bin show very weak winds and very little evolution of both the median $\lambda$ and $\tau$ values.

We note that galaxies characterized by $M_{\star}<10^{10} M_{\odot}$ at redshifts $z<2.2$ maintain their stellar mass below this value during their entire evolution up to redshift $z=0.1$, as visible in Figs. 2 and 3.

Figure 5 shows that the low edge and the median of the infall timescale distribution decrease with decreasing redshift in the two stellar mass bins. This reflects that at each redshift, the galaxies that are on the local MZR and MS with short timescales of accretion $\tau$ and/or high loading factors did not yet exist at high $z$.

In the lowest mass bin, a decrease in median $\tau$ is accompanied by an increase in median $\lambda$ value. This reflects the fact that a larger amount of mass than in more massive systems needs to be expelled to keep their metallicity at low values and to still have them on the MZR.

The median values of the infall timescale $\tau$ at redshift $z=0.1$ are $6.59 \mathrm{Gyr}$ for galaxies with $M_{\star}<10^{10} M_{\odot}$ and $7.25 \mathrm{Gyr}$ for $M_{\star}>10^{10} M_{\odot}$, respectively (see Fig. 5). These values are higher than those found in star-forming galaxies by Spitoni et al. (2017b), where $75 \%$ of the galaxies showed infall timescales shorter than $6 \mathrm{Gyr}$. The difference arises because here we imposed that the galaxies simultaneously follow the MZR and the MSR, whereas in Spitoni et al. (2017b) the only requirement except for the MZR was a specific SFR value above a given 

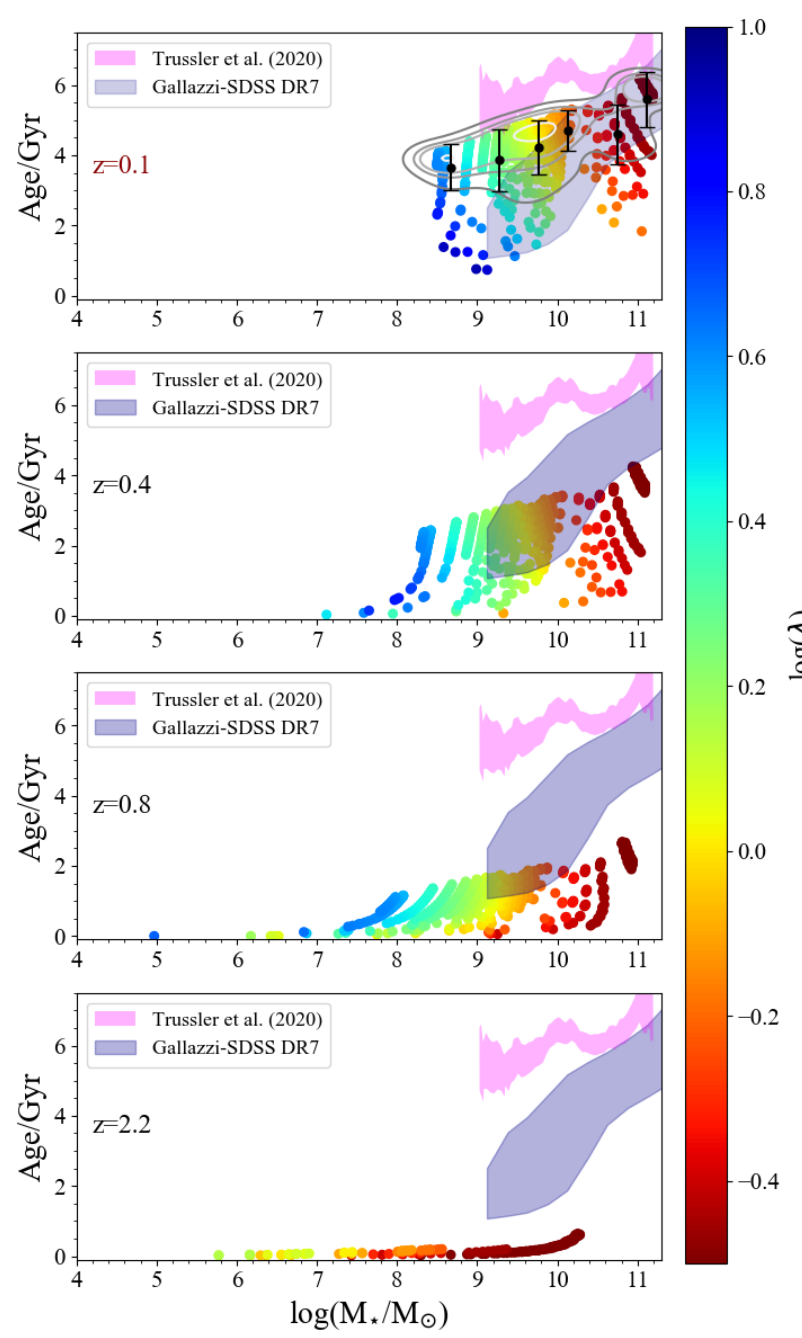

Fig. 6. Backward evolution of the mass-weighted age vs. stellar mass relation (computed at $z=2.2, z=0.8$, and $z=0.4$ ) for the galaxies which lie on the MZR of Kewley \& Ellison (2008) and on the MSR as derived by Peng et al. (2010). The color-coding indicates the loading factor parameter $\lambda$. The white-grey contour lines indicate isodensity contours. The shaded pink area indicates the observational data by Trussler et al. (2020) for local star-forming galaxies $(0.02<z<$ 0.085), whereas the shaded blue area stands for the relation obtained using the SDSS-DR7 catalog of mass-weighted ages estimated as in Gallazzi et al. (2008) using the same selection of star-forming galaxies as Trussler et al. (2020). In the upper panel, the black points are the mean age values of the simulated galaxies at redshift $z \sim 0.1$ inside bins of size $0.5 \log \left(M_{\star} / M_{\odot}\right)$ and the error bars are the standard deviations.

threshold, which was $2.29 \times 10^{-11} \mathrm{yr}^{-1}$. More massive galaxies on the MSR have higher SFRs, and as already mentioned, only these systems can afford the longest accretion timescales, which allow them to exhibit high SFR values down to $z=0.1$.

\subsubsection{Downsizing and scaling relations}

Figure 6 shows the backward evolution of the age versus stellar mass relation for galaxies that at $z \sim 0.1$ obey the MZR and the main sequence of star-forming galaxies. Approximately $89.2 \%$ of the simulated galaxies are located in the region enclosed by the most external contour line in the upper panel of Fig. 6. This positive relation between age and stellar mass is also retained when we increase the resolution of our parameter grid $(\lambda, \tau)$ by
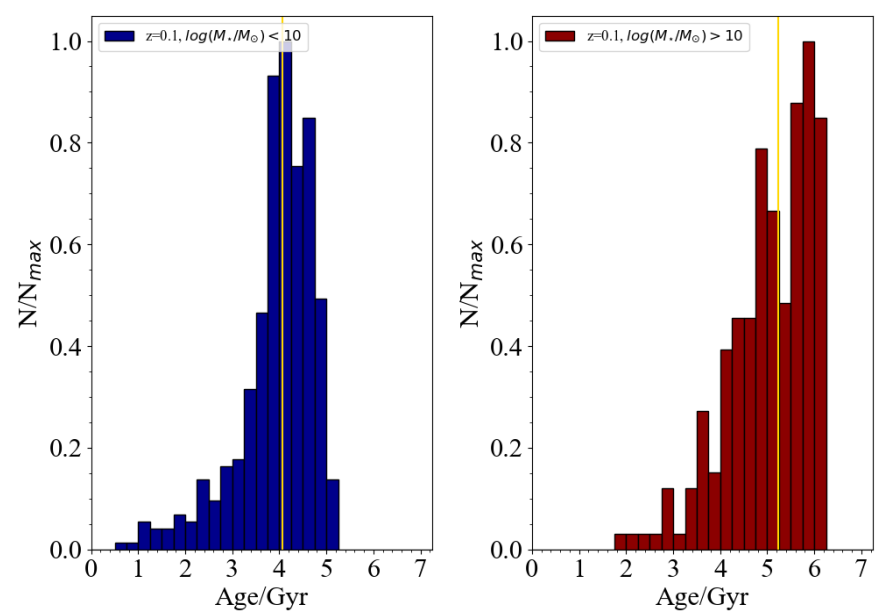

Fig. 7. Age distribution computed at redshift $z=0.1$ for the galaxies which lie on the MZR of Kewley \& Ellison (2008) and on the MSR as derived by Peng et al. (2010) in two different mass bins, $\log \left(M_{\star} / M_{\odot}\right)<$ 10 (left) and $\log \left(M_{\star} / M_{\odot}\right) \geq 10$ (right). In each panel, the yellow vertical line indicates the median value of the distribution.

a factor of two. The positive correlation between age and stellar mass is preserved at any redshift.

From the age distributions computed at redshift $z=0.1$ for the two ranges of stellar masses (see Fig. 7) we infer that the predicted median age of star-forming systems is $\sim 4.06 \mathrm{Gyr}$ (lower stellar mass bin) and 5.23 Gyr (high stellar mass bin). The simulated galaxies at redshift $z=0.1$ reproduce the slope of the observed age-mass relation for star-forming galaxies as found by Trussler et al. (2020). However, the predicted ages are systematically $\sim 1$ Gyr younger than those reported by Trussler et al. (2020).

We also compared the predicted age-mass relation with the relation we obtained adopting the mass-weighted ages estimated as in Gallazzi et al. (2008) for SDSS-DR7 (Gallazzi et al., in prep.; see also Pasquali et al. 2019). From the Gallazzi-DR7 catalog we selected star-forming galaxies with the same criterion as Trussler et al. (2020) and over the same redshift interval. The Gallazzi relation is steeper and overall shifted to lower massweighted ages than the estimates by Trussler et al. (2020).

The noticeable differences in the normalization and slope of the Gallazzi age-mass relation and the Trussler et al. (2020) relation can originate from different assumptions in the observational age estimates: (i) the Bruzual \& Charlot (2003) simple stellar population (SSP) models in comparison to Maraston \& Strömbäck (2011), (ii) the use of a stochastic library of parametric SFHs compared to a linear combination of SSPs without assuming a functional form for the $\mathrm{SFH}$, translating into differences in the allowed fraction of old stars, (iii) limiting the formation age of the models to the age of the Universe compared to allowing SSPs as old as 15 Gyr, and (iv) a Bayesian statistical approach compared to a $\chi^{2}$-minimization code. While it is clear that a quantitative description of the mass-weighted age relation with mass is subject to the observational method adopted, both determinations agree in indicating a positive correlation.

Figure 6 implies a key result: galactic downsizing as reflected from the ages of their stellar populations is naturally accounted for by systems that obey two fundamental scaling relations, namely the MZR and the MSR. When two scaling relations with zero scatter are assumed, together, they predict an age-mass relation with intrinsic scatter $(\sim 0.8 \mathrm{Gyr})$. This means that there 

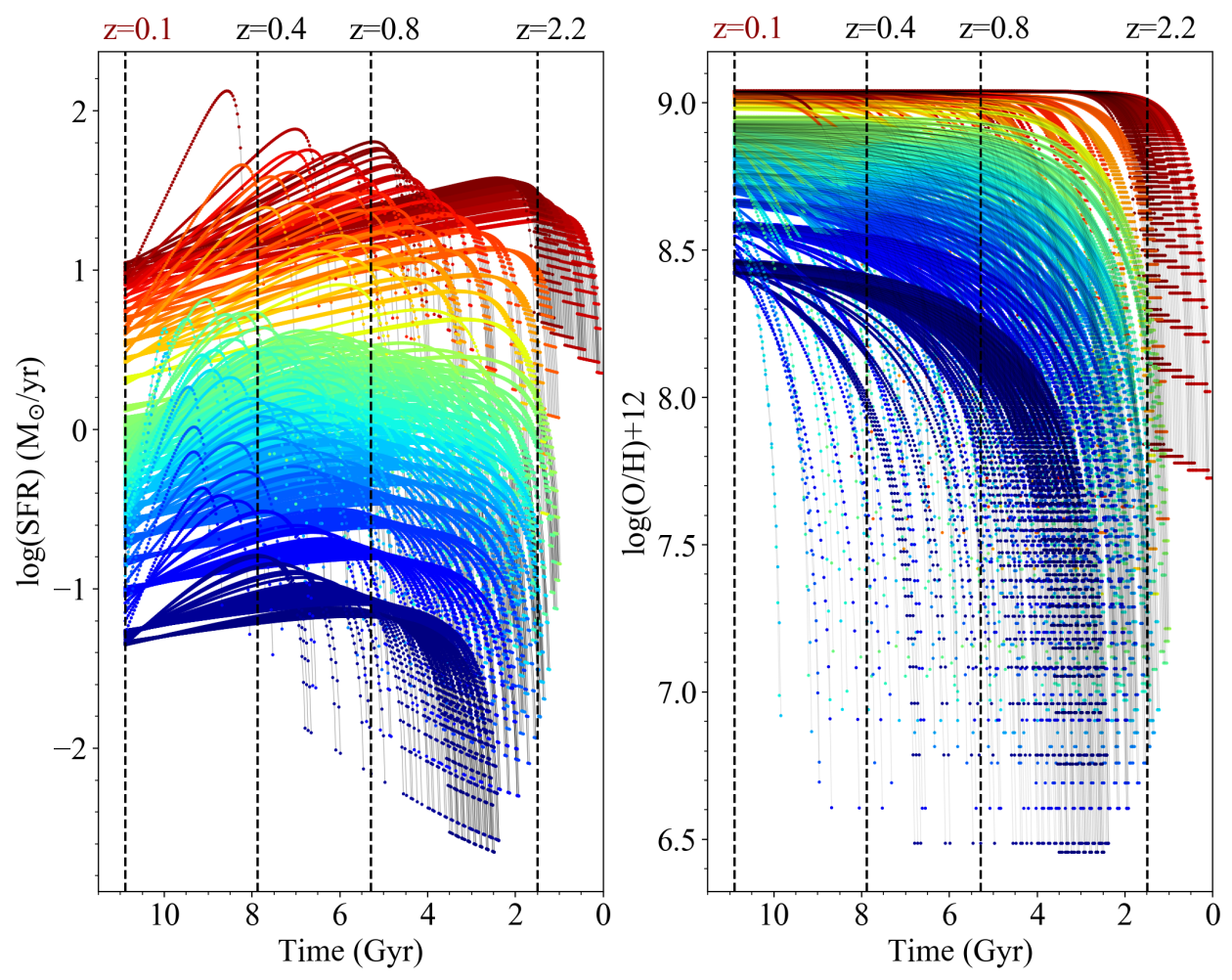

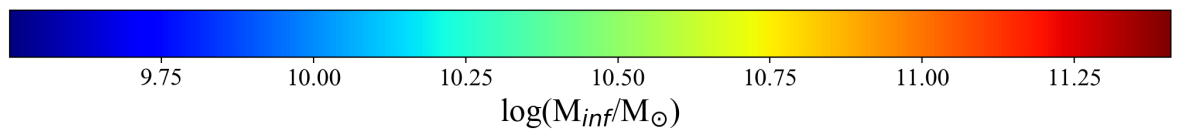

Fig. 8. Evolution of star formation rate (left panel) and oxygen abundance (right panel) as a function of time for the galaxies which lie on the MZR of Kewley \& Ellison (2008) and on the MSR as derived by Peng et al. (2010). The colour coding represents the infall mass. might be a variety of SFHs that can lead to the current equilibrium between SFR and metal enrichment.

The DS of galaxies is also visible from the temporal evolution of the SFR and oxygen abundances of the model galaxies, as shown in Fig. 8. In this figure, the evolution of each quantity is shown for systems of different masses as a function of time for an evolutionary interval of $\sim 10.5 \mathrm{Gyr}$.

These plots show that the bulk of the stars of the galaxies belonging to the highest mass end $\left(\log \left(M_{\text {inf }} / M_{\odot}\right)>11\right)$ of the MZR must have formed at early times. In most of these systems the SFH peaks soon after their formation, and their metallicity saturates at its maximum value $(12+\log (\mathrm{O} / \mathrm{H}) \sim 9)$ after $\sim 1 \mathrm{Gyr}$ of evolution. Some high-mass galaxies may be characterized by SFHs that peak at more recent epochs (i.e., at ages $<4 \mathrm{Gyr}$ ), and present SFR values higher than those of the older galaxies. The systems also have short infall timescales and populate the lowest $\tau-(<6 \mathrm{Gyr})$ tail of the infall timescale distribution. Moreover, they present $\lambda$ values higher than their older analogs, but still $<1$ (see later), implying that galactic outflows are not the main driving mechanisms of their evolution.

Figure 8 shows that the lower the stellar mass of the galaxies, the younger the age at which the SFH peaks and the slower the growth of the metallicity with time. Our analysis of the SFH of these galaxies is in agreement with the one presented in other works (Maiolino et al. 2008; Calura et al. 2009) based on studies of the MZR, although the MZR alone does not necessarily imply galactic downsizing. In order to account for it, we must simultaneously impose MZR and MSR. This represents one main result of our work. With our models, it is also easy to show that the positive correlation between age and mass presented in Fig. 6 disappears completely when we impose that galaxies must follow the MZR alone.
As mentioned in Sect. 3, in our model the temporal evolution of the SFH can be well approximated with a function proportional to $t \mathrm{e}^{-t / \tau}$ (see the discussion in Weinberg 2017). Hence, adopting this analytical expression, the mass-weighted age presented in Eq. (3) computed at the time $t_{n}$ can be rewritten as

$\operatorname{Age}_{\text {approx }}\left(t_{n}\right)=\frac{t_{n}}{1-\mathrm{e}^{-t_{n} / \tau}}-\tau$.

In Fig. 8, the oldest galaxies have been evolving for roughly $t_{n} \simeq 10.5 \mathrm{Gyr}$. Moreover, these objects are characterized by a long accretion timescale $\tau$, and a representative value is $\tau \simeq$ $9.5 \mathrm{Gyr}$ (see Fig. 5). Substituting these quantities in the approximated mass-weighted age introduced above in Eq. (8), we find a value of $\operatorname{Age}_{\text {approx }}\left(t_{n}\right)=6.19 \mathrm{Gyr}$, in perfect agreement with our results for the oldest objects as reported in Fig. 6.

To better understand the main reason for the results discussed here, in Fig. 9 we show the loci occupied by our galaxies in the space of the free parameters of our model, that is, $\lambda$ and $\tau$. The most massive objects are the oldest ones, which are generally located in the bottom right corner of the plots and are characterized by the longest accretion timescales and lowest values for the wind parameter. A population of young very massive systems is visible in Fig. 9, which are the galaxies with the highest SFR values of Fig. 8. In Fig. 9, these systems are considerably underdense with respect to their older analogs. This occurs because in order to follow both the MZR and MSR, only a very limited range of values in the $\lambda-\tau$ space is allowed. In this particular region of this space, small perturbations of the values of each of these parameters are enough to drive galaxies off one (or both) of the two fundamental scaling relations, which explains the paucity of points with $\tau \sim 1$ Gyr and $\lambda \sim 0.8$. On the other hand, a wider range and larger variations for each parameter are 

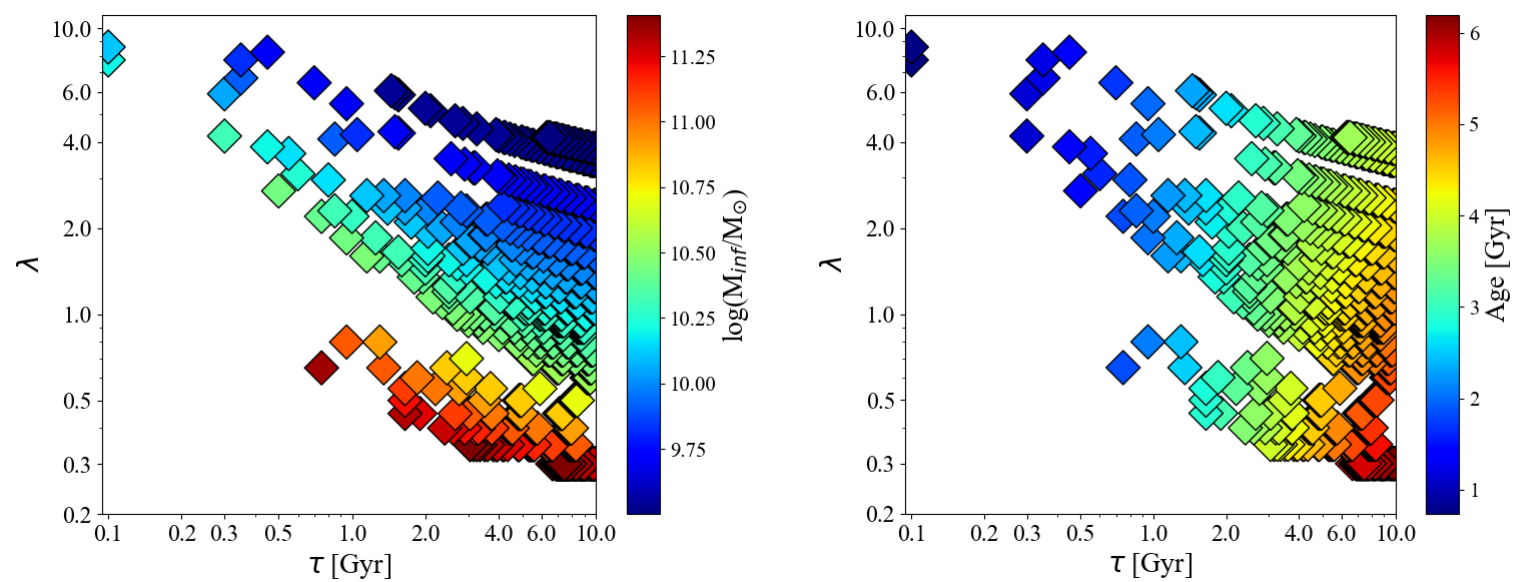

Fig. 9. Parameter space analysis (wind parameter $\lambda$ versus infall time scale $\tau$ ) of the galaxies which line on the MZR of Kewley \& Ellison (2008) and on the main sequence of star-forming galaxies as derived by Peng et al. (2010). In the left panel the colour coding represents the infall mass, whereas in the right one it represents the galactic age.

admitted in the bottom right corner, where massive systems tend to cluster.

In galaxy evolution models, some properties that reflect galactic DS can generally be accounted for in different ways and acting on various parameters, that is, by varying the stellar IMF (Calura \& Menci 2009), or by varying the SFE in galaxies of various mass (Matteucci 1994). In the Sect. 5.1.4 we discuss the role of this parameter in particular further, together with the implications on our results.

\subsubsection{Fundamental metallicity relation of Mannucci et al.} (2010)

Mannucci et al. (2010) showed that star-forming galaxies lie on a tight surface in a 3D space defined by stellar mass, gas-phase metallicity, and SFR. The median values of metallicity of the SDSS galaxies can be expressed by means of a second-order polynomial fit in $M_{\star}$ and SFR:

$$
\begin{aligned}
12+\log (\mathrm{O} / \mathrm{H})= & 8.90+0.37 m-0.14 s-0.19 m^{2} \\
& +0.12 m s-0.054 s^{2},
\end{aligned}
$$

where $m=\log \left(M_{\star} / M_{\odot}\right)-10$ and $s=\log (\mathrm{SFR})$. In our study we analyzed only a subsample of those local SDSS galaxies that, by construction, lie at redshift $z \sim 0.1$ in a curve in this 3D space that is represented by the combination of the MSR and MZR.

However, we can test whether the ancestors of these galaxies lie on the hypersurface defined by the fundamental metallicity relation (FMR). The Mannucci et al. (2010) relation has been defined as "fundamental", implying that in principle, it has to be valid at all redshifts.

In Figs. 10 and 11 we compare in the 3D space defined by $M_{*}, \mathrm{SFR}$, and metallicity the properties of the model galaxies computed at redshift $z=0.1,0.4,0.8,1.5$, and2.2 with the FMR of Mannucci et al. (2010), color-coded as a function of SFR and redshift, respectively. Figures 10 and 11 show that in this 3D space, our galaxies lie on a surface that agrees reasonably well with the FMR determined by Mannucci et al. (2010).

\subsubsection{Downsizing and the star formation efficiency}

In this section we discuss the robustness of our results in accounting for galactic downsizing with respect to the SFE, which is one key parameter of chemical evolution models. As discussed in Sect. 1, an increase in SFE as a function of mass is often invoked in chemical evolution models to explain galactic DS, in particular, as traced by the integrated stellar $[\alpha / \mathrm{Fe}]$ abundance of the stellar populations of local elliptical galaxies (Matteucci 1994; Pipino \& Matteucci 2011).

As introduced in Sect. 3, we assumed the relation of Boselli et al. (2014) between SFE and galaxy mass, where in our case the stellar mass was replaced by the infall mass $M_{\text {inf }}$ (as done also in Spitoni et al. 2017b). However, this assumption is not key for our results, in particular, as far as the capability of our models to account for the age versus stellar mass relation is concerned. To show this, we present the results obtained by adopting a constant $\mathrm{SFE}=1 \mathrm{Gyr}^{-1}$, that is, independent of the mass of the galaxy. In the right panel of Fig. 1, we show the SFE as a function of the infalling gas mass used here that was adapted from the Boselli et al. (2014) scaling relation compared to a constant value of $\mathrm{SFE}=1 \mathrm{Gyr}^{-1}$. Figure 1 shows that the variable $\mathrm{SFE}$ spans a wide range, between 0.01 and $4.5 \mathrm{Gyr}^{-1}$. The relation of Boselli et al. (2014) presents SFE $1 \mathrm{Gyr}^{-1}$ at mass values $\sim 10^{10.5} M_{\odot}$, with a factor $\sim 4.5$ variation between the value at this mass up to the one of galaxies ten times more massive. We also note that low-mass systems can have SFE values that are even lower by a few orders of magnitude.

In Fig. 12 we present the galactic age-stellar mass relation at different redshifts computed for the galaxies that at redshift $z=$ 0.1 are part of the star-forming main sequence and of the MZR, but assuming a constant SFE of $1 \mathrm{Gyr}^{-1}$. This value is typical for chemical evolution models of Milky Way-like galaxies.

A clear DS in the galaxy models is still evident even when a constant SFE is assumed in that on average, the higher the stellar mass, the older their stellar populations. This confirms that our main result, namely that galaxies that lie on both the MZR and MSR are also necessarily characterized by a downsizing in their stellar populations, is independent of the adopted SFE. To our knowledge, this is the first time that this result is derived theoretically.

\subsubsection{Eeffect of a fully $T_{\mathrm{e}}$-based abundance scale for galaxies}

Curti et al. (2017) presented new empirical calibrations for strong-line diagnostics of gas-phase metallicity in local starforming galaxies by uniformly applying the $T_{\mathrm{e}}$ method over the 

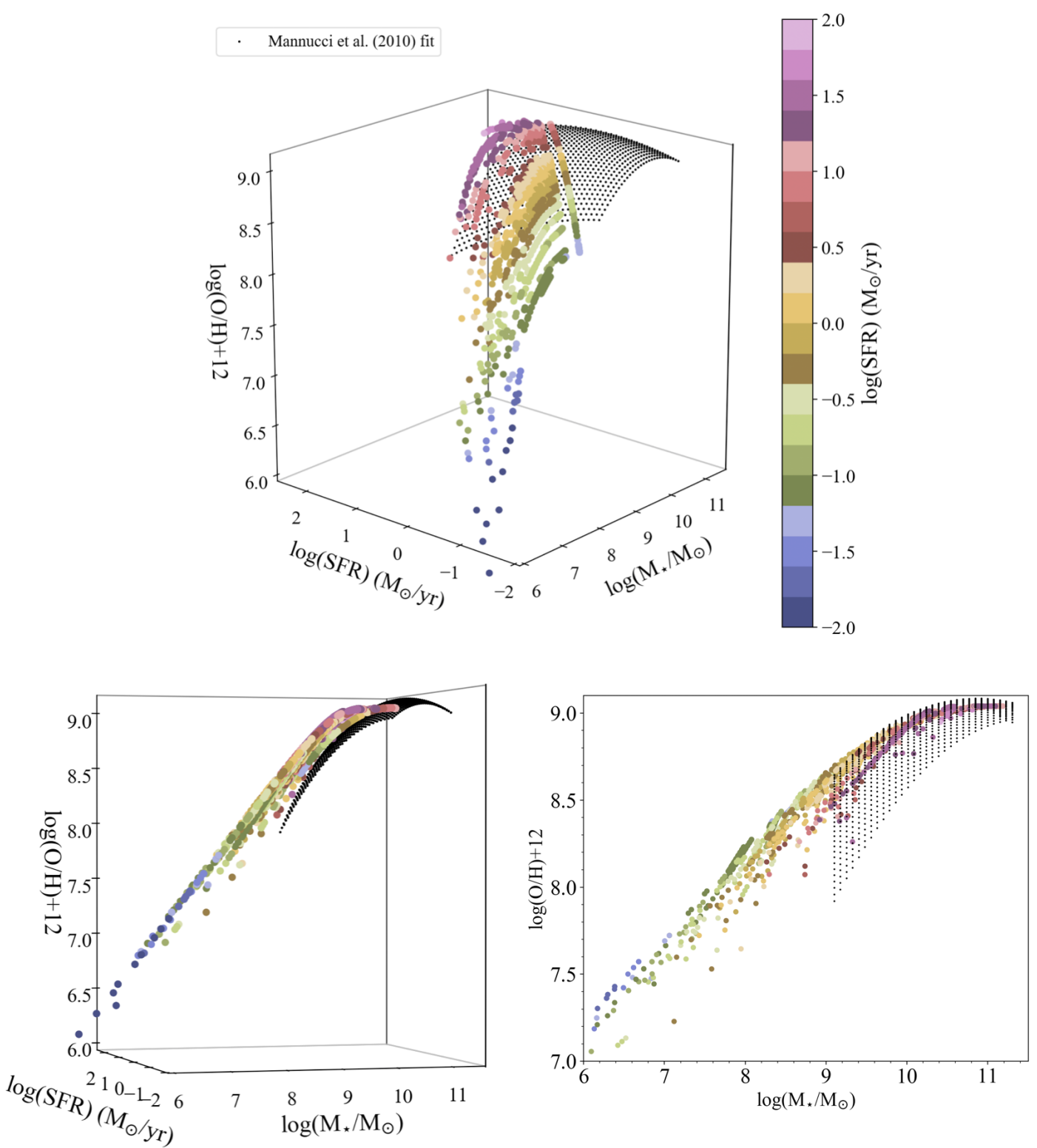

Fig. 10. Three projections of the FMR of Mannucci et al. (2010) compared with the properties of the ancestors of the galaxies that at $z=0.1$ follow the MSR and MZRs. The big solid circles are the model galaxies illustrated in Sect. 5.1.1, and for which the SFR, stellar mass and metallicity values reported in the plots are computed at $z=0.1,0.4,0.8,1.5$ and 2.2, colour-coded as a function of their SFR values. The small black dots are a second-order fit of the SDSS data as presented in Mannucci et al. (2010).

full metallicity range probed by the SDSS. Moreover, an MZR for local SDSS galaxies obtained with the $T_{\mathrm{e}}$-based method was presented.

The new fit of the median MZR for the SDSS sample is

$12+\log (\mathrm{O} / \mathrm{H})=Z_{0}-\gamma / \beta * \log \left(1+\left(\frac{M_{\star}}{M_{0}}\right)^{-\beta}\right)$,

where $Z_{0}=8.793, \log \left(M_{0} / M_{\odot}\right)=10.02, \gamma=0.28$, and $\beta=1.2$. From the left panel of Fig. 1, it is clear that the slope and overall normalization of the MZR are sensitive to the calibration method (see also Kewley \& Ellison 2008), and that the relation obtained by Curti et al. (2017) is flatter and generally characterized by lower metallicity values than that of Kewley \& Ellison (2008).

In this section, we test whether the assumption of a purely $T_{\mathrm{e}}$-based MZR also affects the main results presented above. Figures 13-15 show results that are qualitatively similar to those of
Figs. 2, 4 and 6, respectively. The MZR of Curti et al. (2020) produces stronger winds than the Kewley \& Ellison (2008) MZR (Figs. 13 and 14). This result can be ascribed to the lower oxygen abundances of the galaxies that follow the Curti et al. (2020) MZR, as in the framework of the present work, it is by means of stronger winds that galaxies can have a lower metal content than the models computed when the Kewley \& Ellison (2008) MZR was assumed. Figure 15 also shows that galactic downsizing is preserved when the Curti et al. (2020) MZR is used, with a predicted age-mass relation very similar to that of Fig. 6.

\subsection{Forward evolution}

\subsubsection{MZR by Maiolino et al. (2008) and MSR at $z=2.2$}

We are also interested in the evolution of galaxies that at high redshift are part of both the main sequence and of the MZR. 

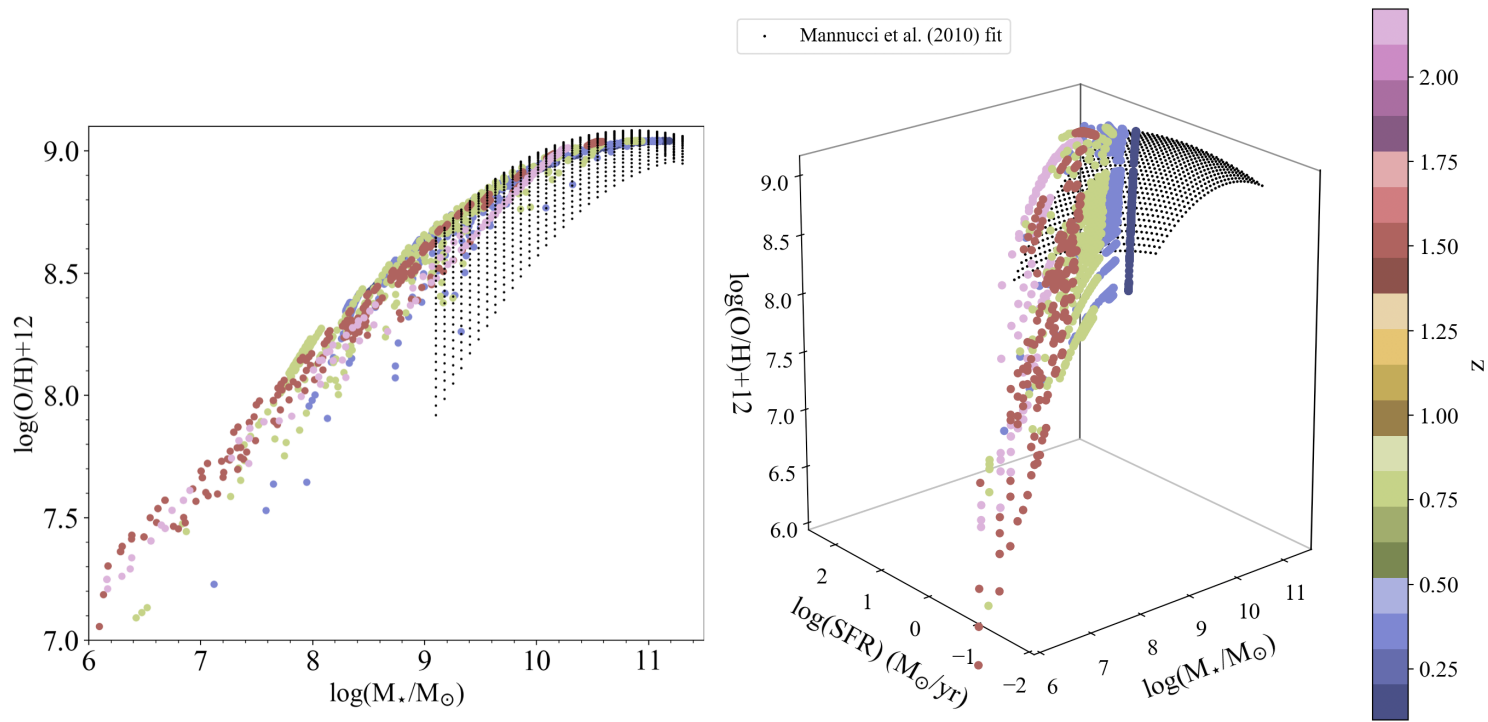

Fig. 11. Two projections of the FMR of Mannucci et al. (2010) compared with the properties of the model galaxies as in Fig. 10, colour-coded as a function of their redshift $z$.
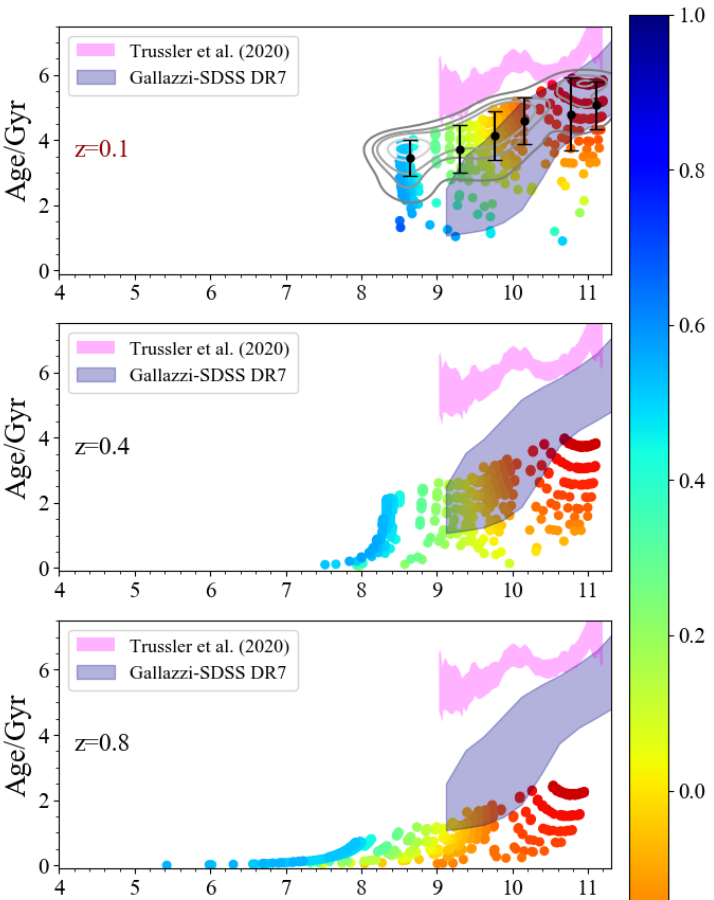

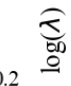

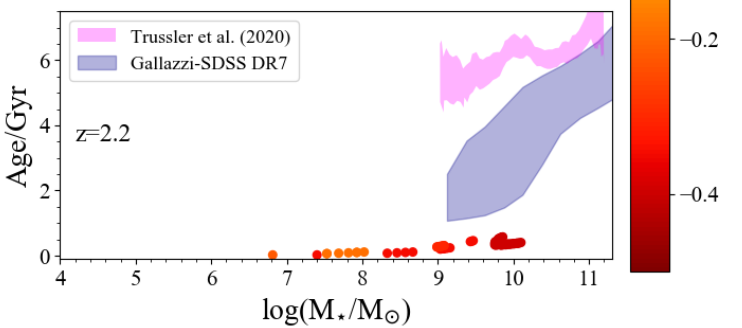

Fig. 12. As in Fig. 6, but computed assuming a constant SFE fixed at the value of $1 \mathrm{Gyr}^{-1}$.

As for the MZR, the observational constraint we discuss here is the one by Maiolino et al. (2008), and the star-forming MSR is that by Pearson et al. (2018) for galaxies observed in the redshift range $1.8 \leq z \leq 2.3$.
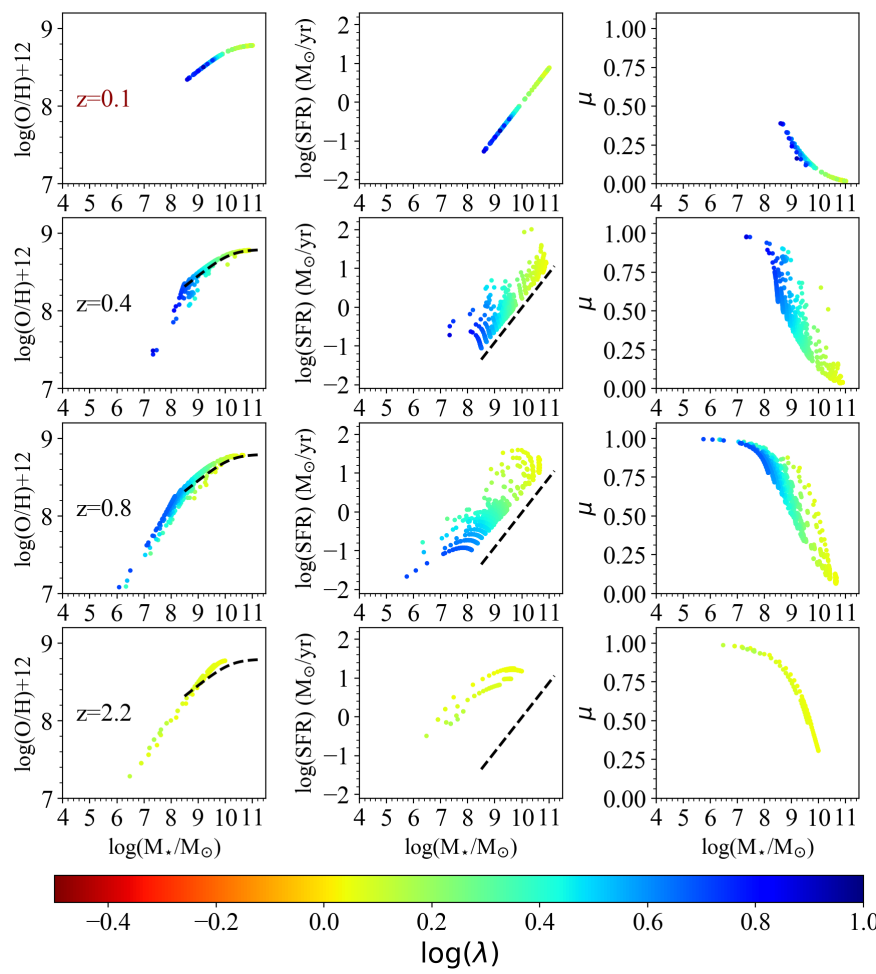

Fig. 13. Backward evolution from $z=0.1$ to $z=2.2$ of the galaxies that obey the analytical fit of the observed MZR at $z \sim 0.1$ of Curti et al. (2020) and the local main sequence of star-forming galaxies as derived by Peng et al. (2010), color-coded as a function of the loading factor parameter $\lambda$ as in Fig. 2. The dashed black lines in the panels of the first column and in each SFR- $\log \left(M_{\star} / M_{\odot}\right)$ plot show the MZR of Curti et al. (2020) and the MSR derived in local star-forming galaxies by Peng et al. (2010), respectively.

In the middle panel of Fig. 1 we compare the local MSR with that at high redshift by Pearson et al. (2018). Large differences characterize the two sequences, in particular, as far as the zeropoint of the relation is concerned. An offset by at least one order of magnitude in SFR is visible, for instance, at stellar masses 

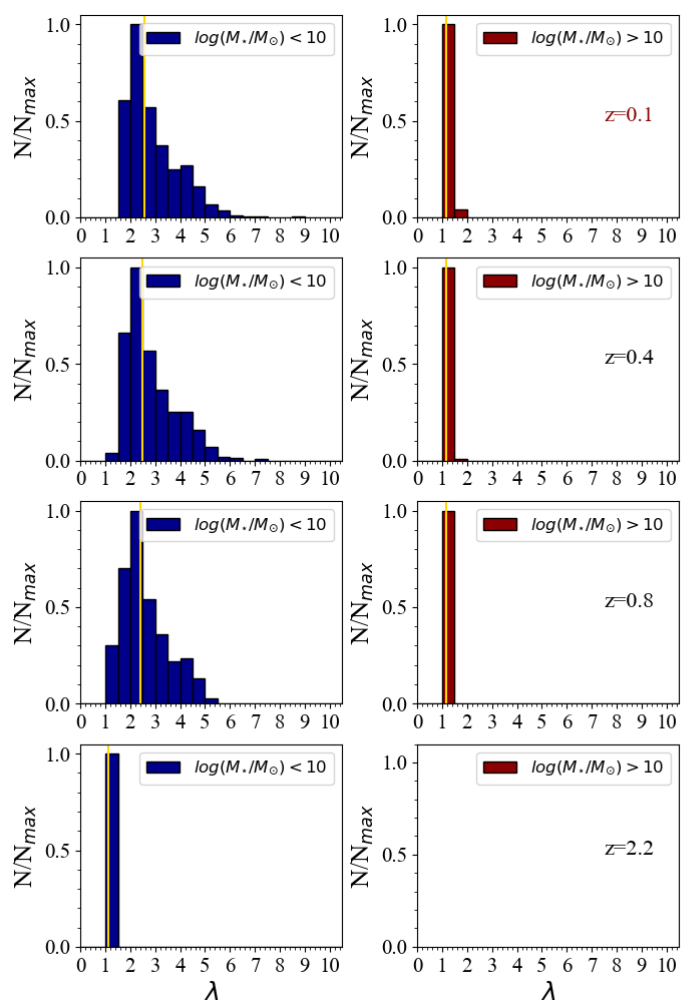

Fig. 14. As in Fig. 4, but computed with the local MZR proposed by Curti et al. (2020).

$\log \left(M_{\star} / M_{\odot}\right) \sim 11$, whereas any variation in the slope is much less appreciable. This difference in normalization is expected from the evolution of the cosmic star formation rate density (e.g., Madau \& Dickinson 2014) and comparable to what was found in other studies (e.g., Santini et al. 2017). The MZR considered in this section (solid thick red line in the left panel of Fig. 1) is steeper than the local one (solid thick blue line), and as discuss by Maiolino et al. (2008), this might imply different evolutionary timescales in galaxies of different masses.

In Fig. 16 we show the forward (i.e., evolved toward lower redshifts, corresponding to later epochs) evolution of the galaxies that at $z=2.2$ obey the MZR and MSR, computed at $z=2.2$, $z=0.8, z=0.4$, and $z=0.1$.

The high SFR values required by the high-redshift MSR and the associated low oxygen abundances of the MZR relation force the galactic systems to be subject to stronger winds and assembled on shorter timescales than the galaxies presented in Sect. 5.1.1 (see Fig. 17). Hence, galaxies suffer strong depletion of gas and evolve passively at redshifts $z<2$.2. Figure 16 shows that the gas fraction drops dramatically from redshift $z=2.2$ to redshift $z=0.8$. The most massive objects show high stellar masses (built up in a short time) and high metallicity and must have consumed their reservoir of gas soon after redshift $z=2.2$. After this, the buildup of their stellar mass and metals was already complete, and they have undergone little evolution at later times. Less massive galaxies, characterized by stronger winds (see Fig. 17), present a more sensible evolution, which manifests in a steepening of the MZR toward recent times. In conclusion, the forward evolution of the single galaxies in the MZR relation is characterized by a horizontal evolution at roughly constant metallicity.

The passive evolution of these galaxies can also be inferred by their age-stellar mass relation at redshift $z=0.1$, which
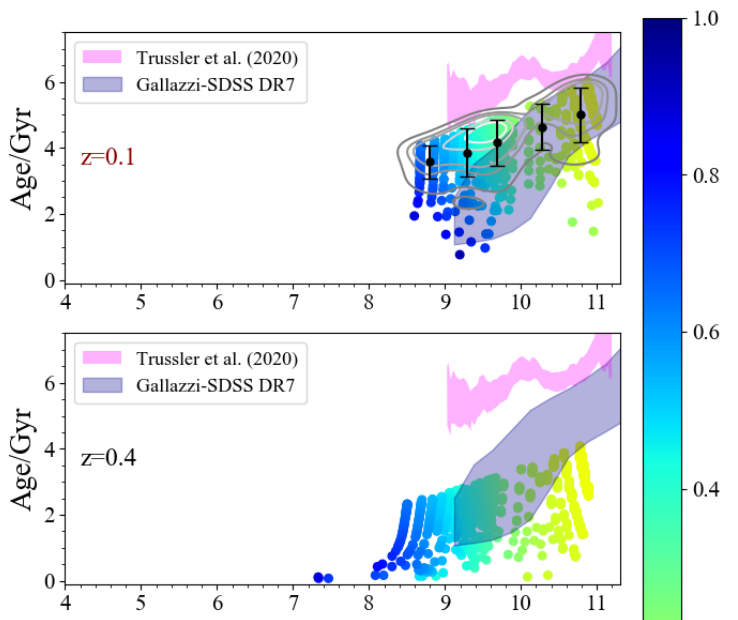

0.6
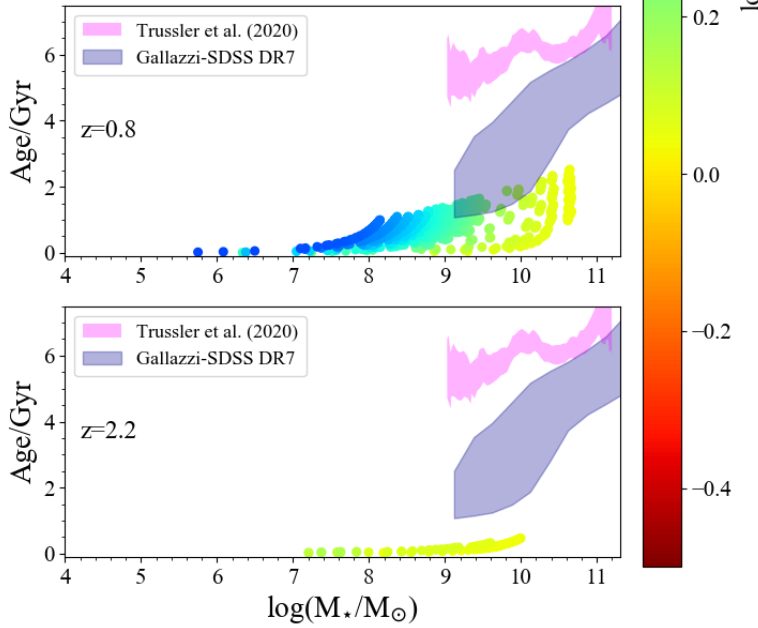

Fig. 15. As in Fig. 6, but computed assumingthe MZR proposed by Curti et al. (2020) at redshift $z \sim 0.1$.

is similar to that followed by local passive galaxies of Trussler et al. (2020). However, passive galaxies in Trussler et al. (2020) have much higher stellar metallicities than simulated galaxies at redshift $z=0.1$. In principle, these galaxies could have been part of the local passive sequence of Trussler et al. (2020) as due to a quenching mechanism, such as the one proposed by Peng et al. (2015): for example, by strangulation (see their lower panel in Fig. 1). After the beginning of strangulation, no outflow and no gas accretion affect the galactic system, and the star formation can continue with the gas available in the galaxy until it is completely used up. During this phase, the gas metallicity increases substantially because of the lack of dilution from inflowing gas and gas loss.

As already underlined by Maiolino et al. (2008), galaxies belonging to the MZR observed at different redshifts should not be seen as evolutionary sequences. Figure 17 clearly shows that at redshift $z=2.2$, these galaxies are characterized by stronger winds than the galaxies studied in Sect. 5.1.1. This is a result of imposing that they also belong to the MSR, which at $z=2.2$ is characterized by higher SFR values than the one at low redshift in the entire stellar mass range. In order to simultaneously present low metallicities and high SFR values, it is clear that galaxies at $z=2.2$ must have suffered much stronger stellar winds than galaxies belonging to the MZR and MSR at lower redshifts.

In Fig. 18 we compare the properties of the galaxies that at redshift $z \sim 2.2$ follow the MZR and MSR relations with the 

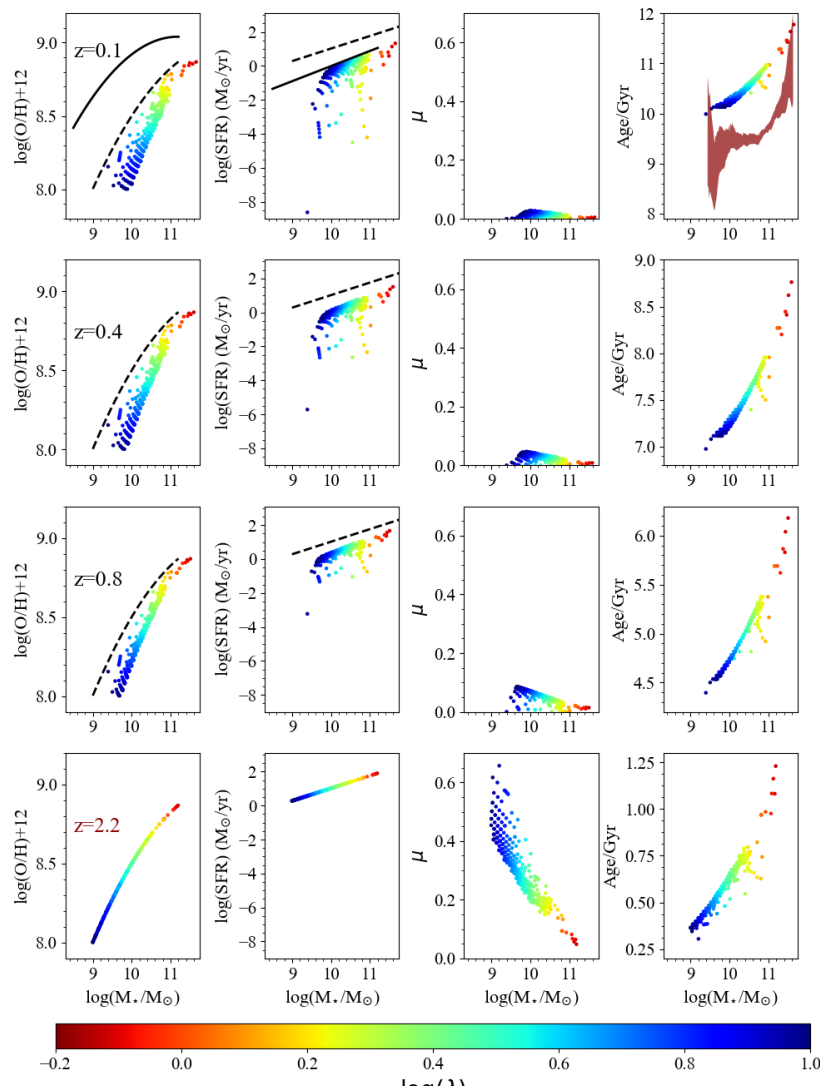

$\log (\lambda)$

Fig. 16. Forward evolution from $z=2.2$ to $z=0.1$ of the galaxies that obey the analytical fit of the observed MZR at $z \sim 2.2$ by Maiolino et al. (2008) and the main sequence of star-forming galaxies as derived by Pearson et al. (2018). Starting from the left, in the first, second and third column we show the evolution of the MZR, SFR vs. stellar mass and gas fraction vs stellar mass, respectively. In the last column, the age-stellar mass relation is shown. The color-coding indicates the loading factor parameter $\lambda$. The black dashed lines in the panels of the first column and in each SFR- $\log \left(M_{\star} / M_{\odot}\right)$ plot, show the MZR at redshift $z \sim 2.2$ of Maiolino et al. (2008) and the MSR derived in high redshift star forming galaxies by Pearson et al. (2018), respectively. The solid black lines in the first two top-left panels are the MZR at redshift $z \sim 0.1$ of Kewley \& Ellison (2008) and the MSR of Peng et al. (2010), respectively. In the upper-right panel with the dark red shaded area we also include the age-stellar mass relation for the local passive galaxies by Trussler et al. (2020).

FMR of Mannucci et al. (2010). The models shown in Fig. 18 are computed at redshifts $z=0.1,0.4,0.8$, and 2.2 and are colorcoded as a function of $z$. The loci occupied by the model galaxies partially overlap the FMR only at $z=2.2$, whereas at lower redshifts, they fall off the surface traced by the black points, which represents the analytical fit to the FMR presented in Sect. 5.1.3. The reason is that galaxies that at $z=2.2$ follow both MZR and MSR become passive at later times, as shown by the progressive decline of their SFR values.

\subsubsection{MZR by Mignoli et al. (2019) and MSR at $z=2.2$}

In the narrow line regions (NLR) of a sample of $88 \mathrm{C}$ IVselected objects containing type 2 AGNs, Mignoli et al. (2019) measured at $z \sim 2.2$ a nearly flat MZR, set at the value of $12+\log (\mathrm{O} / \mathrm{H}) \sim 8.5$ dex. It is worth mentioning that the computed metallicity of the NLR in the selected type 2 AGNs is
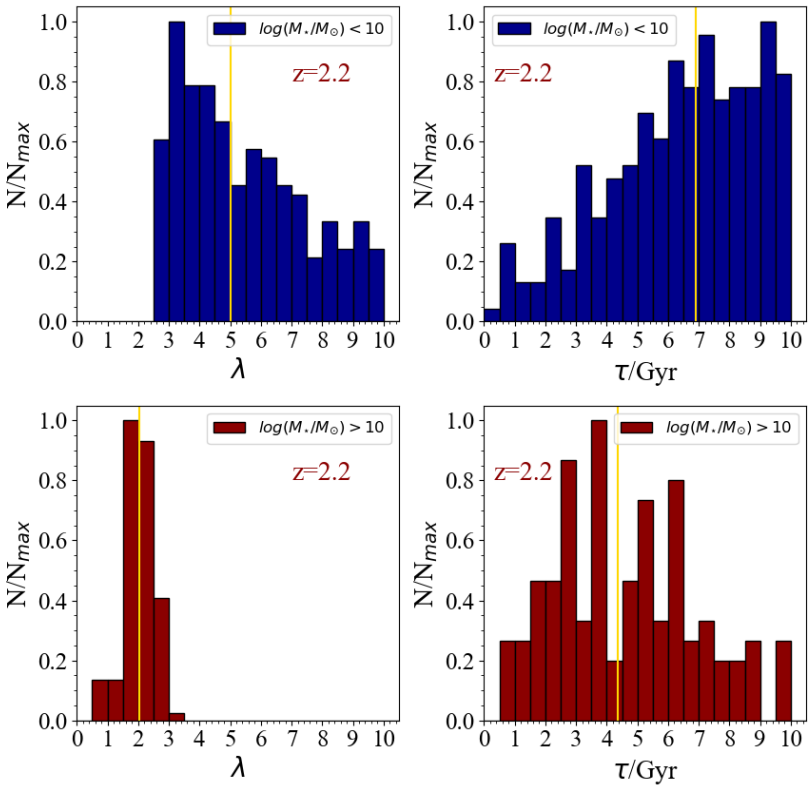

Fig. 17. Distribution of the predicted galaxies of Fig. 16 in terms of the loading factor parameter $\lambda$ (left panels) and the infall time-scale parameter $\tau$ (right panels), computed at redshift $z=2.2$ for different stellar mass bins.

based on calibrations from photoionization models of various rest-UV diagnostic line ratios. These methods are quite different from those adopted in normal star-forming galaxies as in Maiolino et al. (2008), which involve rest-optical lines and a combination of $T_{\mathrm{e}}$-measurements and photoionization modeling. The comparison between the two should therefore be made with caution.

As suggested by Mignoli et al. (2019), one of the possible reasons for a flat $\mathrm{MZ}$ relation is a selection effect in the sample. The high-ionization C IV emission line can cause a culling of massive and dust-free hosts that are not representative of the starforming galaxy population at these redshifts. Another possibility is that the metallicity of the NLR is not a good proxy of that of the host galaxy because an AGN can strongly affect not only the ionization properties of the gas, but also the circulation of metals in the ISM, for example, by means of a strong outflow.

We hypothesize that the galaxy sample of Mignoli et al. (2019) includes average star-forming galaxies. By means of our models, we computed the forward evolution of the galaxies that at $z=2.2$ belong to the star-forming MSR of Pearson et al. (2018) and also present a flat MZR as observed by Mignoli et al. (2019), as shown in Fig. 19. We used the model with a constant SFE, fixed at the value of $1 \mathrm{Gyr}^{-1}$ as discussed earlier. Figure 19 shows that the required infall masses are higher than the galaxies that belong to the local MZR and the MSR (see Fig. 3).

A high oxygen abundance has to be already in place at high redshift even for galaxies with low stellar masses, and high infall masses are naturally accompanied by high metal production. As for high stellar mass systems, the MSR imposes high SFR values $\left(1<\log \left(\mathrm{SFR} / M_{\odot}, \mathrm{yr}^{-1}\right)<2\right)$. In order to maintain a high star formation level, large reservoirs of gas (i.e., high infall masses) are required.

Figure 19 shows that the mass-weighted ages versus stellar mass relation (computed at different redshifts) shows a larger spread compared the relation presented in Fig. 16, where at high redshift the MZR of Maiolino et al. (2008) was imposed. We verified that this difference is mainly due to the fact that in the 

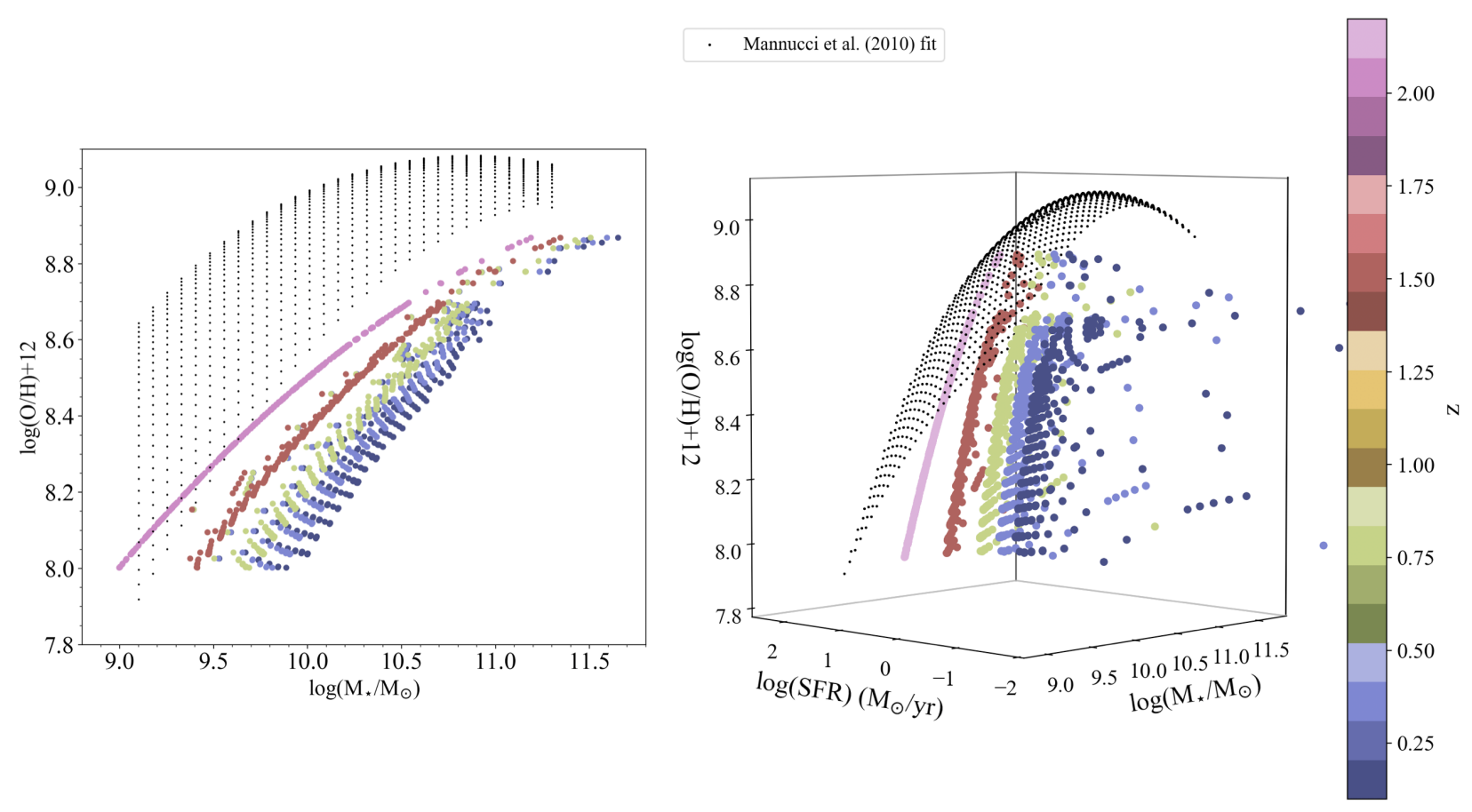

Fig. 18. As in Fig. 11 but for galaxies which at $z=2.2$ follow the MZR of Maiolino et al. (2008) and the MSR derived by Pearson et al. (2018). The big solid circles are computed at redshifts $z=2.2, z=1.5, z=0.8, z=0.4$, and $z=0.1$ and are colour-coded as a function of the redshift value. Black dots are as in Fig. 11.

last case, galaxies with the same infalling mass present a similar $\mathrm{SFH}$, and consequently, they have the same mass-weighted age. In contrast, the high-redshift flat MZR leads to a wide range of $\mathrm{SFH}$ at a fixed value for the infall mass in the successive evolution phases.

In their subsequent evolution, the galaxies settle onto an inverted MZR, as already visible at $z=1$. This occurs because owing to their high SFR values, the system with the highest stellar masses soon become passive, hence their metal content does not change significantly at later times.

Low-mass galaxies are characterized by lower SFR values and by an already high metallicity. Their low SFR values and their relatively high infall masses allow them to maintain their star formation activity and to continue producing new metals, with an unavoidable consequent increase in their metallicity. In this case, their winds are not sufficient to cause a significant decrease in their metallicity and to have them settled on a normal MZR at lower redshifts.

These results might indicate that either our hypothesis that these galaxies are on the MSR is inadequate, or that one fundamental ingredient is missing in our models that causes a substantial redistribution of metals, such as the feedback of an AGN. At present, investigations are ongoing in order to constrain the SFR of these systems and to assess which region they occupy in the SFR- $M_{*}$ diagram, to be compared to the MSR of normal star-forming galaxies observed at $z \sim 2$.

In Fig. 20 we explore the evolution of the stellar metallicity for the galaxies of the Mignoli et al. (2019) flat MZR. This quantity can be computed from our models in the following way:

$\left\langle Z_{\star}(t)\right\rangle=\frac{\int_{0}^{t} \mathrm{~d} t^{\prime} Z\left(t^{\prime}\right) \psi\left(t^{\prime}\right)}{\int_{0}^{t} \mathrm{~d} t^{\prime} \psi\left(t^{\prime}\right)}$.

Equation (10) is by definition the mass-weighted stellar metallicity, that is, the mass-weighted average of the metallicity of the stellar populations in each single galaxy (see Pagel 1997). It is interesting to note that at redshift $z=2.2$, the shape of the stellar MZR is very different from the flat relation of the gas-phase metallicity. The stellar MZR presents more of a standard form, with an increasing behavior at the low-mass edge and a flattening at $\log \left(M_{*} / M_{\odot}\right) \sim 10$. This is a consequence of having imposed the MSR and arises because more massive objects have suffered on average stronger SF episodes at their earliest stages.

However, this relation is much flatter than the relations discussed previously in Sect. 5.2.1, as the variation between the metallicity of the lowest mass galaxies with $\log \left(M_{*} / M_{\odot}\right) \sim 8.5$ and the plateau is of only $0.02 \mathrm{dex}$. At redshift $z=0.1$, also the stellar metallicity presents an inverted shape, the with highest metallicity values at the lowest masses, as already seen in Fig. 19. In summary, in our view, the galaxies of a sample like the one of Fig. 19 that build up a flat MZR do not represent an average sample of star-forming galaxies at high redshift, which are expected to follow a steep MZR, as other works have shown (Erb et al. 2006; Maiolino et al. 2008). The sample of Fig. 19, in which all galaxies include a type 2 AGN, is likely to be composed of systems at various evolutionary stages, in which the AGN played an important role in regulating the star formation activity.

As a final note, another work in which the MZR in galaxies hosting type 2 AGN is the one of Matsuoka et al. (2018). Their sample includes high- $z$ radio galaxies and X-ray selected radio-quiet AGNs at $1.2<z<4.0$ with stellar mass values $>10^{10} M_{\odot}$. In this mass interval, Matsuoka et al. (2018) derived a MZR consistent with that of Maiolino et al. (2008). However, due to the selection of their sample, their study does not probe the low-mass end of the MZR. In the future, metallicity measures of more extended datasets of AGN hosts will be needed to shed more light on the evolution of their MZR, and to further highlight possible differences with the scaling relations of star-forming galaxies. 

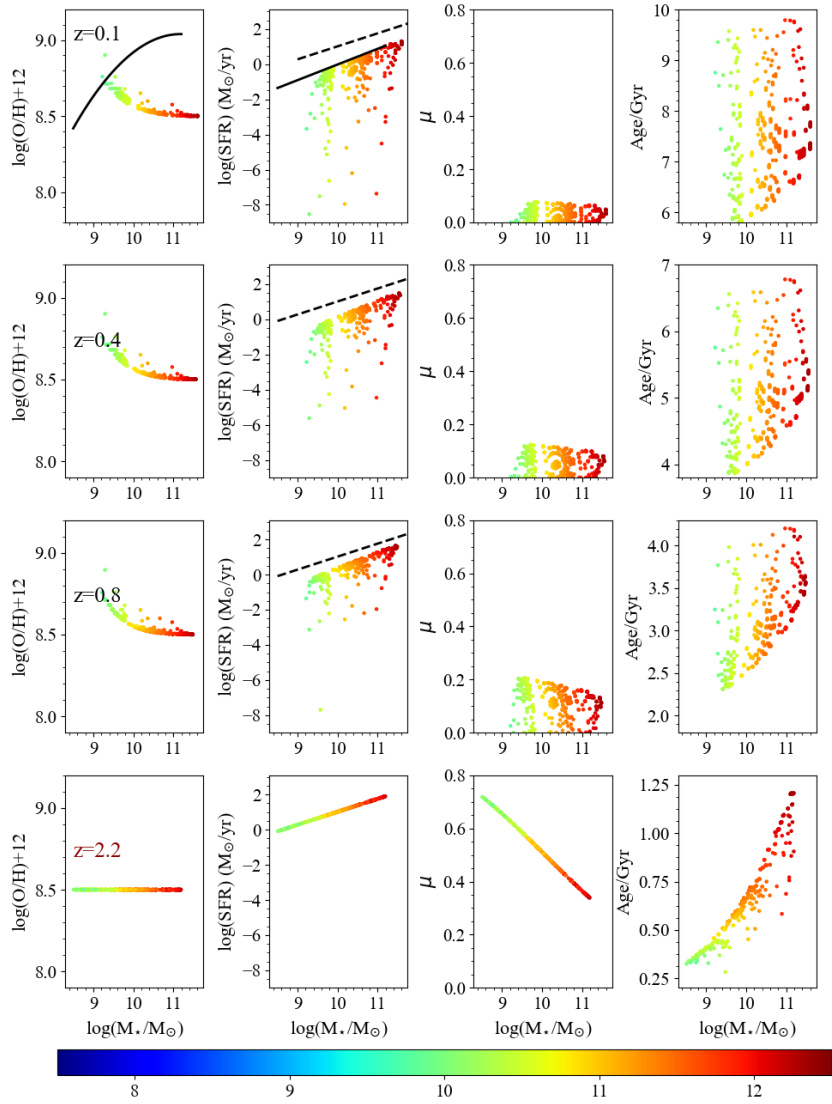

$\log \left(\mathrm{M}_{\mathrm{inf}} / \mathrm{M}_{\odot}\right)$

Fig. 19. Forward evolution from $z=2.2$ to $z=0.1$ of the galaxies that obey the analytical fit of the MZR at $z \sim 2.2$ observed by Mignoli et al. (2019) and the main sequence of star-forming galaxies as derived by Pearson et al. (2018). Starting from the left, in the first, second, third, and fourth column the evolution of the MZR, SFR vs. stellar mass, gas fraction vs. stellar mass and age-stellar mass relation are shown, computed at redshifts $z=2.2, z=0.8, z=0.4$, and $z=0.1$ (from bottom to top) The color-coding stands for the infall gas-mass value. The thick solid black lines in the first two top left panels are the MZR at redshift $z \sim 0.1$ of Kewley \& Ellison (2008) and the MSR of Peng et al. (2010), respectively. The dashed black lines in each SFR- $\log \left(M_{\star} / M_{\odot}\right)$ plot stand for the MSR derived in high-redshift star-forming galaxies by Pearson et al. (2018).

\section{Conclusions}

We have studied the evolution of the systems that follow two fundamental observed scaling relations for star-forming galaxies: the MZR and the MSR. Our study was performed by means of the analytical leaky-box chemical evolution model of Spitoni et al. (2017b). In this model, galaxies are assumed to be formed by means of accretion of primordial gas, with an accretion rate that follows an exponential law, and in presence of galactic winds. In the model, each galaxy is characterized in terms of three model-free parameters: the total infalling mass $M_{\text {inf }}$, the loading factor wind parameter $\lambda$, and the timescale of gas accretion $\tau$.

We focused on a given redshift, and we imposed that the galaxies must follow the observed MZR and the star-forming MSR measured at that particular redshift. First, we studied the properties of the local galaxies by imposing the MZR and the MSR observed at redshift $z=0.1$, and analyzing their backward evolution toward higher redshifts. On the other hand, by imposing the same scaling relations at higher redshift, we computed a
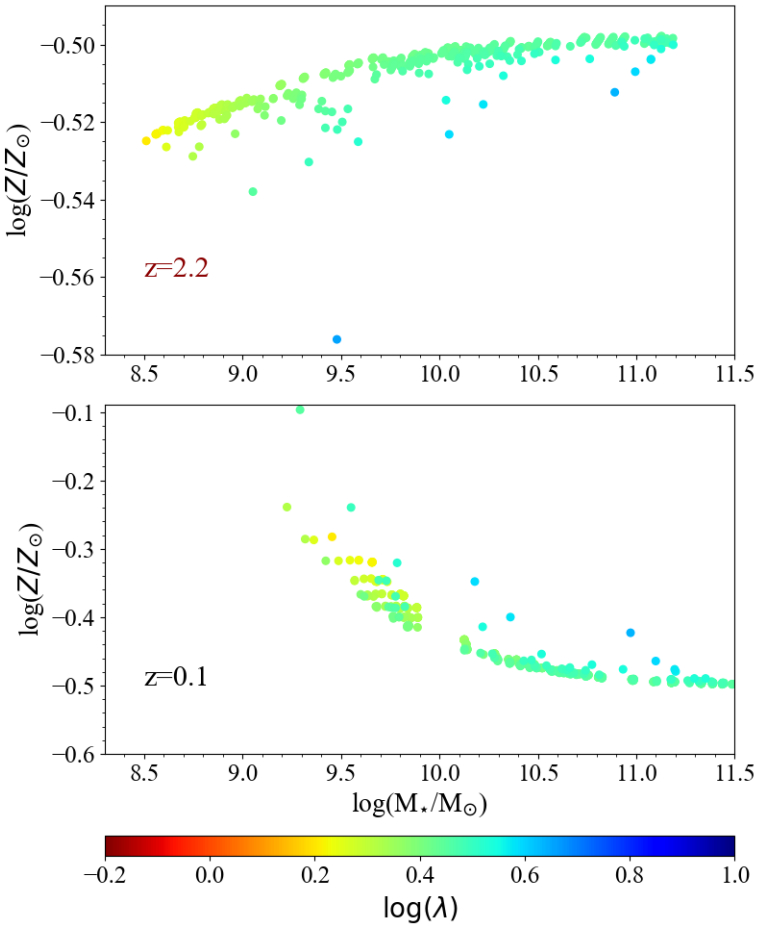

Fig. 20. Stellar mass-metallicity relation for the galaxies that follow the Mignoli et al. (2019) MZR and the high-redshift star-forming main sequence by Pearson et al. (2018) computed at redshift $z=2.2$ (upper panel) and $z=0.1$ (lower panel).

forward evolution of the galaxies at lower redshifts and toward the present time. Our main conclusions are summarized below.

- By imposing the MZR and the MSR at redshift $z=0.1$, we showed that the galaxies already present at redshift $z=2.2$ are characterized by weak winds and long timescales of gas accretion. At low redshift, these objects have to maintain a large reservoir of gas in order to be part of the local MSR and a high metallicity, as they now build the highmetallicity plateau of the MZR. Moreover, the median of the infall timescale distribution in two different bins of stellar masses $\left(M_{\star}<10^{10} M_{\odot}\right.$ and $\left.M_{\star}>10^{10} M_{\odot}\right)$ decreases with decreasing redshift. This reflects that at each redshift, only the youngest galaxies can be assembled on the shortest timescales and still belong to the star-forming MSR. In the lowest mass bin, a decrease in median $\tau$ is accompanied by an increase in median $\lambda$ value. This implies that systems that have formed at more recent times will need to eject more mass to maintain their metallicity at low values.

- Imposing both the MZR and the MSR naturally leads to an increasing relation between the mass-weighted age and the stellar mass, which is qualitatively in agreement with the relation observed in local SF galaxies obtained with independent determinations. We note, however, that a quantitative agreement is difficult to assess because different observational estimates of the age-mass relation are sensitive to the assumption on SFH, SSP models, and technique used. To our knowledge, such a direct connection between the MZR, MSR, and downsizing has not been shown before in any theoretical study. Moreover, no successful attempt has been performed so far to simultaneously reproduce these fundamental scaling relations within a cosmological framework, mostly because of an early overproduction of low-mass galaxies, which in such models are currently 
dominated by stellar populations that are too old (Calura et al. 2014; Somerville \& Davé 2015).

- In a 3D space formed by SFR, oxygen abundances, and stellar mass, the ancestors of the galaxies that follow the observed MZR and the star-forming MSR at $z=0.1$ (as studied at $z \leq 2.2$ ) lie on a hyper-surface, which is in good agreement with the FMR of Mannucci et al. (2010).

- We studied the evolution of galaxies that at high redshift are part of both the observed MSR and of the MZR. By imposing at redshift $z=2.2$ the MZR of Maiolino et al. (2008), we found that the high SFR values required by the high-redshift MSR force most of the galaxies to evolve passively at redshifts lower than $z=2.2$. The most massive objects show high stellar masses (built up in a short time) and high metallicity and must have consumed their reservoir of gas soon after leaving the MZR. Less massive galaxies, characterized by stronger winds, present a slower evolution, which manifests in a progressive steepening of the MZR toward recent times. In order to simultaneously present low metallicities and high SFR values, it is clear that galaxies at $z=2.2$ must have suffered much stronger stellar winds than galaxies belonging to the MZR and MSR at lower redshifts. Therefore, our findings confirmed that the observed MZRs and MSRs at different redshifts should not be interpreted as evolutionary sequences (see also Maiolino et al. 2008 and Lian et al. 2018a).

- In our analysis, we also calculated the forward evolution of high-redshift objects that at $z \sim 2$ are characterized by a flat MZR, as observed by Mignoli et al. (2019). The sample by Mignoli et al. (2019) includes galaxies with a type 2 AGN and selected from their C IV emission and for which the star formation rate is unknown. In this particular case, even lowmass galaxies have to present a high metallicity already at early times. In this case, the system with the highest stellar masses furthermore become soon passive, whereas lower mass galaxies can still keep their star formation activity, with a further increase in their metallicity and despite their winds, which, even if stronger than more massive systems, are not sufficient to significantly deplete their metal reservoirs. The implication is that in their subsequent evolution, the galaxies settle onto an inverted MZR. These results might indicate that one fundamental ingredient is missing in our model, which causes a substantial redistribution of metals within each single galaxy, such as the feedback of an AGN. Another possibility is that at present, these systems do not follow the MSR. At present, investigations are ongoing in order to constrain the SFR of these systems and to assess which region they occupy in the SFR- $M_{\star}$ diagram.

Acknowledgements. The authors thank the referee for the useful comments, which have certainly improved the paper. We thank G. Zamorani for useful suggestions. Funding for the Stellar Astrophysics Centre is provided by The Danish National Research Foundation (Grant agreement no.: DNRF106). E. Spitoni also acknowledges funding from the INAF-OAS visitor program. E. Spitoni and V. Silva Aguirre acknowledge support from the Independent Research Fund Denmark (Research grant 7027-00096B). V. Silva Aguirre acknowledges suppor from VILLUM FONDEN (Research Grant 10118). FC acknowledges support from grant PRIN MIUR 2017 - 20173ML3WW_001 and from the INAF MainStream (1.05.01.86.31)

\section{References}

Boselli, A., Cortese, L., Boquien, M., et al. 2014, A\&A, 564, A66 Brinchmann, J., Charlot, S., White, S. D. M., et al. 2004, MNRAS, 351, 1151 Bruzual, G., \& Charlot, S. 2003, MNRAS, 344, 1000

Calura, F., \& Menci, N. 2009, MNRAS, 400, 1347
Calura, F., Jimenez, R., Panter, B., Matteucci, F., \& Heavens, A. F. 2008, ApJ, 682,252

Calura, F., Pipino, A., Chiappini, C., Matteucci, F., \& Maiolino, R. 2009, A\&A, 504,373

Calura, F., Menci, N., \& Gallazzi, A. 2014, MNRAS, 440, 2066

Chabrier, G. 2003, PASP, 115, 763

Cowie, L. L., Songaila, A., Hu, E. M., \& Cohen, J. G. 1996, AJ, 112, 839

Curti, M., Cresci, G., Mannucci, F., et al. 2017, MNRAS, 465, 1384

Curti, M., Mannucci, F., Cresci, G., \& Maiolino, R. 2020, MNRAS, 491, 944

Elbaz, D., Daddi, E., Le Borgne, D., et al. 2007, A\&A, 468, 33

Erb, D. K., Steidel, C. C., Shapley, A. E., et al. 2006, ApJ, 647, 128

Gallazzi, A., Charlot, S., Brinchmann, J., White, S. D. M., \& Tremonti, C. A. 2005, MNRAS, 362, 41

Gallazzi, A., Brinchmann, J., Charlot, S., \& White, S. D. M. 2008, MNRAS, 383, 1439

Geha, M., Blanton, M. R., Masjedi, M., \& West, A. A. 2006, ApJ, 653, 240

Grisoni, V., Spitoni, E., \& Matteucci, F. 2018, MNRAS, 481, 2570

Hirschmann, M., De Lucia, G., \& Fontanot, F. 2016, MNRAS, 461, 1760

Iyer, K., Gawiser, E., Davé, R., et al. 2018, ApJ, 866, 120

Kannappan, S. J. 2004, ApJ, 611, L89

Kewley, L. J., \& Dopita, M. A. 2002, ApJS, 142, 35

Kewley, L. J., \& Ellison, S. L. 2008, ApJ, 681, 1183

Köppen, J., Weidner, C., \& Kroupa, P. 2007, MNRAS, 375, 673

Larson, R. B. 1974, MNRAS, 169, 229

Lequeux, J., Peimbert, M., Rayo, J. F., Serrano, A., \& Torres-Peimbert, S. 1979, A\&A, 500, 145

Lian, J. H., Li, J. R., Yan, W., \& Kong, X. 2015, MNRAS, 446, 1449

Lian, J., Thomas, D., Maraston, C., et al. 2018a, MNRAS, 474, 1143

Lian, J., Thomas, D., \& Maraston, C. 2018b, MNRAS, 481, 4000

Madau, P., \& Dickinson, M. 2014, ARA\&A, 52, 415

Maiolino, R., \& Mannucci, F. 2019, A\&ARv, 27, 3

Maiolino, R., Nagao, T., Grazian, A., et al. 2008, A\&A, 488, 463

Mannucci, F., Cresci, G., Maiolino, R., Marconi, A., \& Gnerucci, A. 2010, MNRAS, 408, 2115

Maraston, C., \& Strömbäck, G. 2011, MNRAS, 418, 2785

Matsuoka, K., Nagao, T., Marconi, A., et al. 2018, A\&A, 616, L4

Matteucci, F. 1994, A\&A, 288, 57

Matteucci, F. 2012, Chemical Evolution of Galaxies (Berlin, Heidelberg: Springer-Verlag )

Mignoli, M., Feltre, A., Bongiorno, A., et al. 2019, A\&A, 626, A9

Mortlock, A., Conselice, C. J., Bluck, A. F. L., et al. 2011, MNRAS, 413, 2845

Noeske, K. G., Weiner, B. J., Faber, S. M., et al. 2007, ApJ, 660, L43

Pagel, B. E. J. 1997, Nucleosynthesis and Chemical Evolution of Galaxies (Cambridge, UK : Cambridge University Press)

Pasquali, A., Smith, R., Gallazzi, A., et al. 2019, MNRAS, 484, 1702

Pearson, W. J., Wang, L., Hurley, P. D., et al. 2018, A\&A, 615, A146

Peng, Y., Maiolino, R., \& Cochrane, R. 2015, Nature, 521, 192

Peng, Y.-J., Lilly, S. J., Kovač, K., et al. 2010, ApJ, 721, 193

Pérez-Montero, E., Contini, T., Lamareille, F., et al. 2013, A\&A, 549, A25

Pipino, A., \& Matteucci, F. 2011, A\&A, 530, A98

Recchi, S., Spitoni, E., Matteucci, F., \& Lanfranchi, G. A. 2008, A\&A, 489, 555

Romano, D., Karakas, A. I., Tosi, M., \& Matteucci, F. 2010, A\&A, 522, A32

Salpeter, E. E. 1955, ApJ, 121, 161

Santini, P., Fontana, A., Castellano, M., et al. 2017, ApJ, 847, 76

Savaglio, S., Glazebrook, K., Le Borgne, D., et al. 2005, in The Spectral Energy Distributions of Gas-Rich Galaxies: Confronting Models with Data, eds. C.

C. Popescu, R. J. Tuffs, et al., Am. Inst. Phys. Conf. Ser., 761, 425

Schmidt, M. 1959, ApJ, 129, 243

Somerville, R. S., \& Davé, R. 2015, ARA\&A, 53, 51

Spitoni, E. 2015, MNRAS, 451, 1090

Spitoni, E., Calura, F., Matteucci, F., \& Recchi, S. 2010, A\&A, 514, A73

Spitoni, E., Vincenzo, F., Matteucci, F., \& Romano, D. 2016, MNRAS, 458, 2541

Spitoni, E., Vincenzo, F., \& Matteucci, F. 2017a, A\&A, 599, A6

Spitoni, E., Gioannini, L., \& Matteucci, F. 2017b, A\&A, 605, A38

Spitoni, E., Cescutti, G., Minchev, I., et al. 2019a, A\&A, 628, A38

Spitoni, E., Silva Aguirre, V., Matteucci, F., Calura, F., \& Grisoni, V. 2019b, A\&A, 623, A60

Spitoni, E., Verma, K., Silva Aguirre, V., \& Calura, F. 2020, A\&A, 635, A58

Spolaor, M., Kobayashi, C., Forbes, D. A., Couch, W. J., \& Hau, G. K. T. 2010, MNRAS, 408, 272

Tremonti, C. A., Heckman, T. M., Kauffmann, G., et al. 2004, ApJ, 613, 898

Trussler, J., Maiolino, R., Maraston, C., et al. 2020, MNRAS, 491, 5406

Vincenzo, F., Matteucci, F., Belfiore, F., \& Maiolino, R. 2016, MNRAS, 455, 4183

Vincenzo, F., Spitoni, E., Calura, F., et al. 2019, MNRAS, 487, L74

Weinberg, D. H. 2017, ApJ, 851, 25

Yuan, T. T., Kewley, L. J., \& Richard, J. 2013, ApJ, 763, 9

Zahid, H. J., Dima, G. I., Kudritzki, R.-P., et al. 2014, ApJ, 791, 130 


\section{Appendix A: Model results with the Chabrier (2003)} IMF

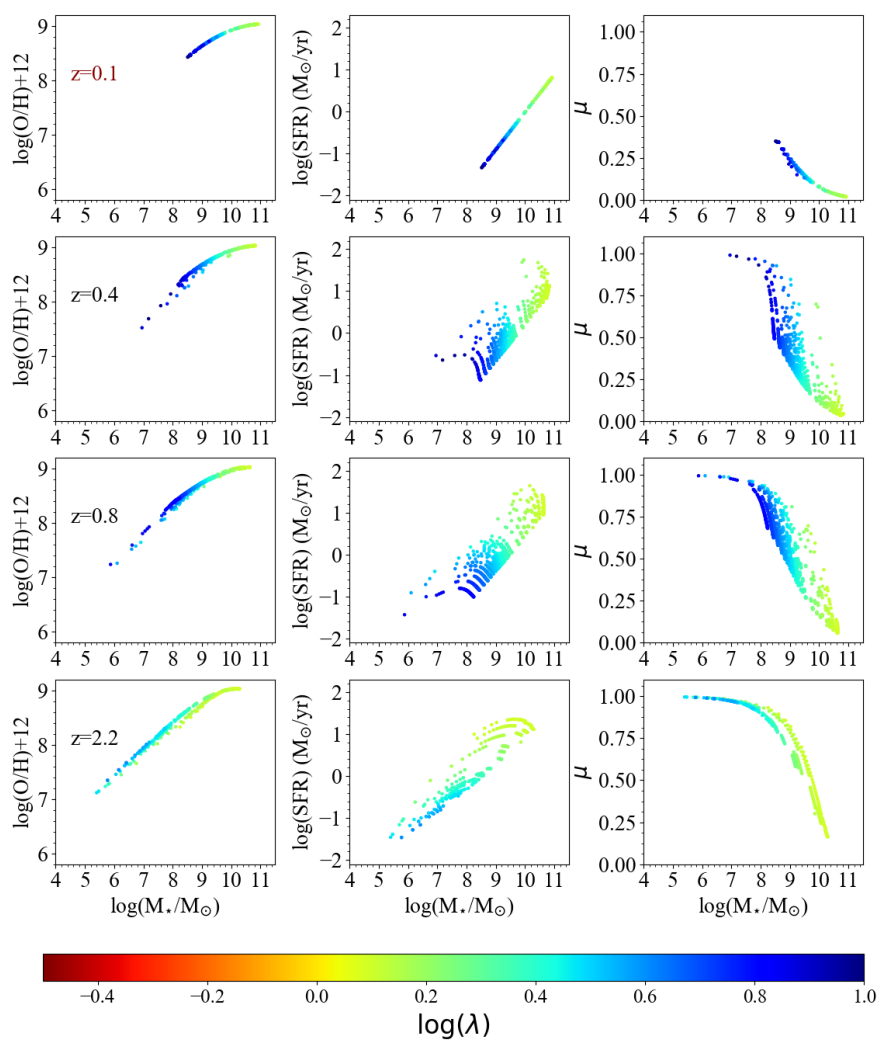

Fig. A.1. Backward evolution from $z=0.1$ to $z=2.2$ of the galaxies that obey the analytical fit of the observed MZR at $z \sim 0.1$ and the local main sequence of star-forming galaxies as in Fig. 2, but computed assuming a Chabrier (2003) IMF. In the first, second, and third column we show the evolution of MZR, SFR vs. stellar mass, and gas fraction vs. stellar mass, respectively. The color-coding indicates the loading factor parameter $\lambda$.

In this appendix we present our model results similar to what we discussed in Sect. 5.1.1, but computed assuming a Chabrier (2003) IMF. We still considered as constraints the observed MZR and star-forming main sequence at redshift $z=0.1$, and we present the backward evolution of some of the properties that build these two relations, including the gas fraction and age-mass relation.

As mentioned in Sect. 2, the Chabrier (2003) IMF is richer in massive stars than the Salpeter IMF. When we assume the compilation of stellar yields presented by Romano et al. (2010), the oxygen yield per stellar generation computed with a Chabrier (2003) IMF is higher by a factor of $\sim 2.3$ than the value computed with the Salpeter (1955) IMF.

In Fig. A.1 we show the backward evolution of the MZR (first column), SFR-mass relation, and gas fraction versus stellar mass relation for galaxies that at redshift $z=0.1$ lie on the MZR and on the main sequence. Compared to the Salpeter (1955) case, in Fig. A.1 we note that the sequence of the first galaxies that have appeared at redshift $z=0.8$ is more populated and more extended toward low stellar mass values. This is an obvious consequence of the higher yield for stellar generation, which leads to a more efficient chemical enrichment in all galaxies, and hence at any time, galaxies with a lower stellar mass can show the same oxygen abundance. Moreover, Fig. A.2 shows that the
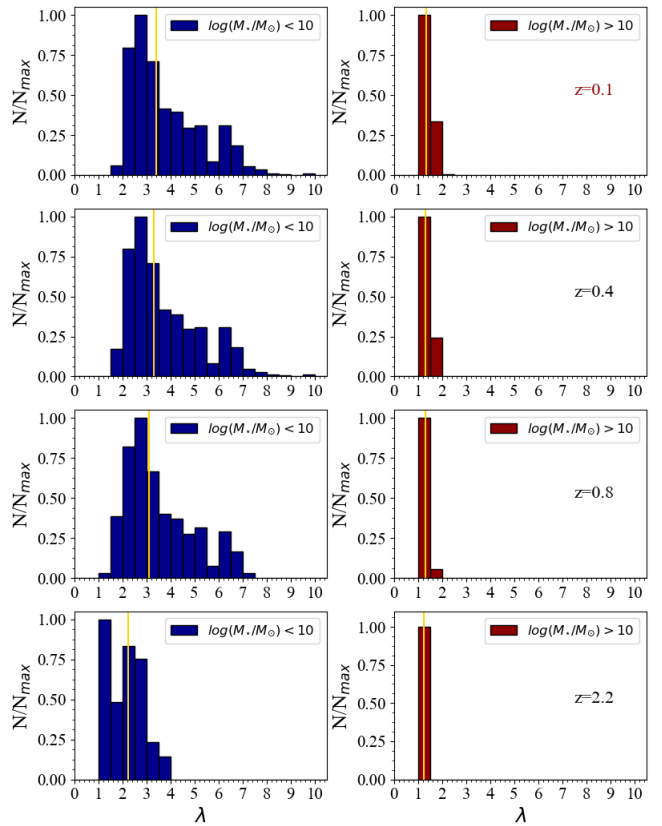

Fig. A.2. Distribution of the loading factor parameter $\lambda$ for the galaxies of Fig. A.1 computed at redshifts $z=2.2, z=0.8, z=0.4$, and $z=$ 0.1 for 2 different bins of stellar mass (left: $\log \left(M_{\star} / M_{\odot}\right)<10$; right: $\left.\log \left(M_{\star} / M_{\odot}\right)>10\right)$. The vertical yellow lines indicate the median values of each distribution.
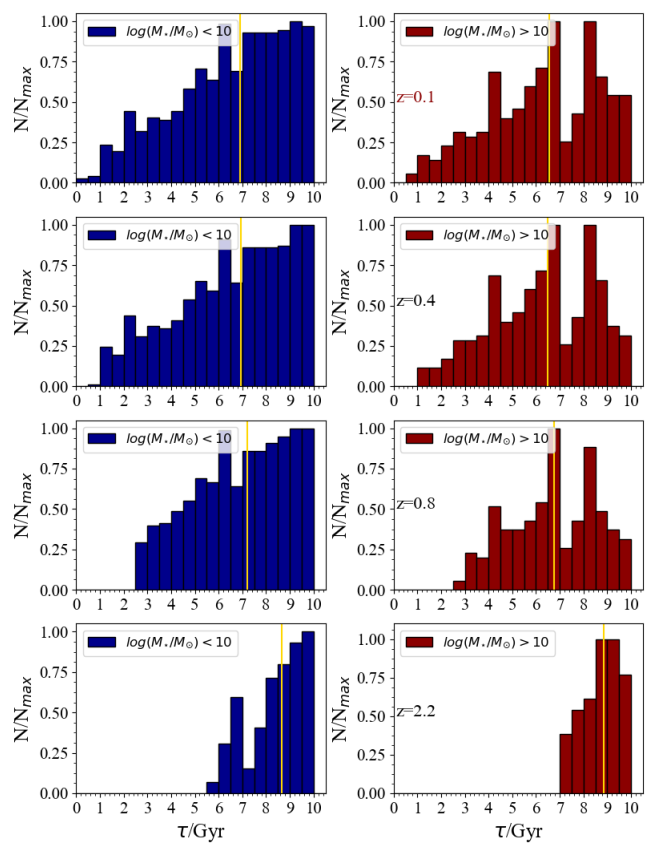

Fig. A.3. As in Fig. A.2, but in each panel the plotted distribution is for the infall timescale parameter $\tau$.

Chabrier (2003) IMF requires stronger winds than the Salpeter (1955) IMF, as reported in Fig. 4, confirming previous results by Spitoni et al. (2017b).

The evolution of the age-mass relation for our galaxies is shown in Fig. A.4, and the predicted age distribution computed at redshift $z=0.1$ in two different redshift bins is shown in Fig. A.5. Both figures show that on average, the Chabrier (2003) IMF produces older galaxies than the Salpeter (1955) IMF. 


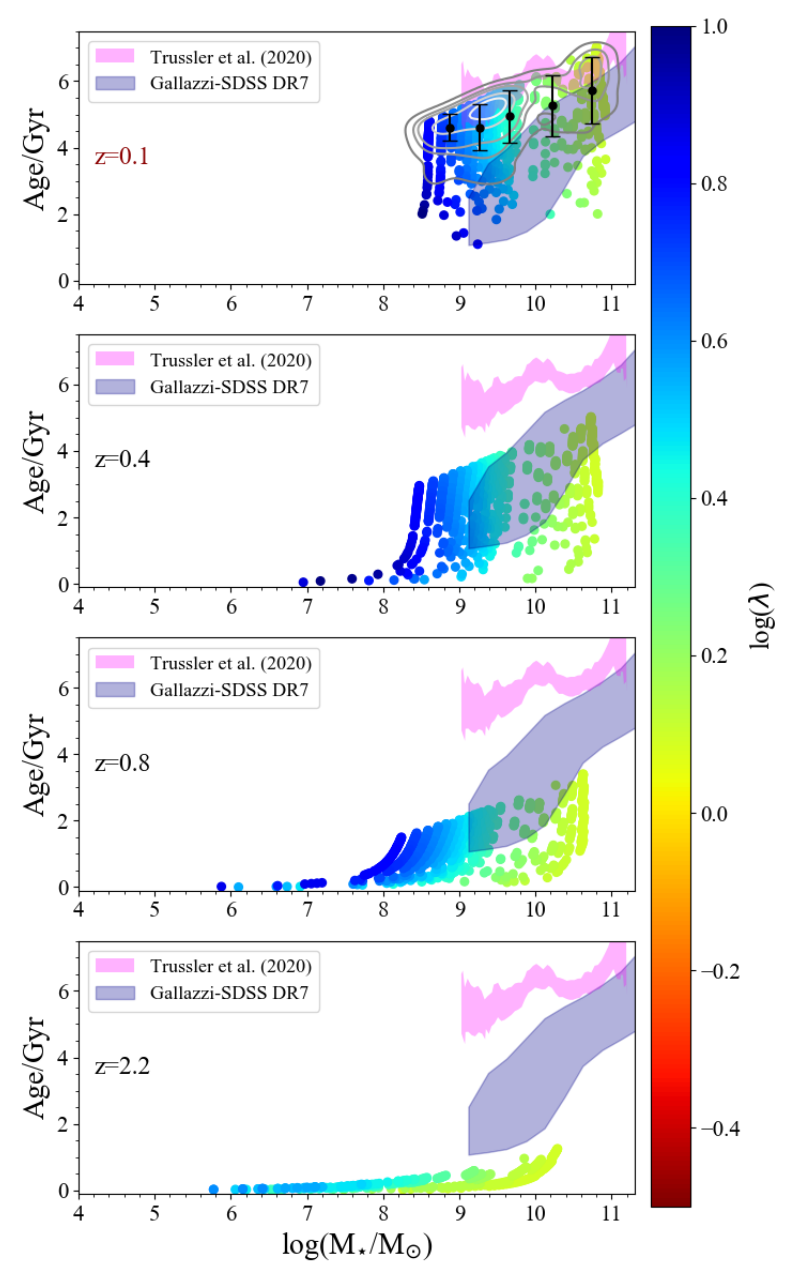

Fig. A.4. Backward evolution of the mass-weighted age vs. stellar mass relation for the galaxies of Fig. A.1, computed adopting a Chabrier (2003) IMF.
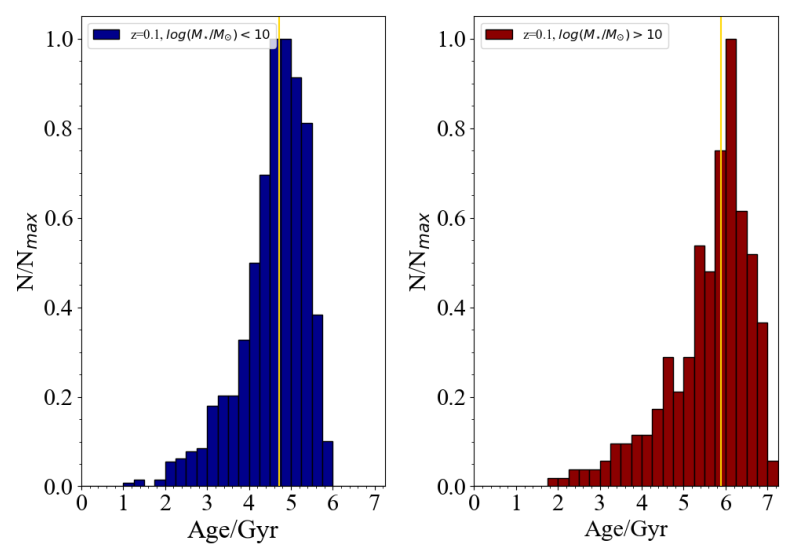

Fig. A.5. Age distribution for star-forming galaxies that are on the MZR at $z=0.1$ in two different mass bins as in Fig. 7, computed assuming a Chabrier (2003) IMF.

This result seems to be in contrast with the results obtained by Spitoni et al. (2017b), in which, by using only the local MZR as a constraint for star-forming galaxies, the Chabrier (2003) IMF produced younger galaxies than the Salpeter (1955) IMF. This difference is only due to the fact that in this case, we also used the MSR as another observational constraint for local starforming systems.

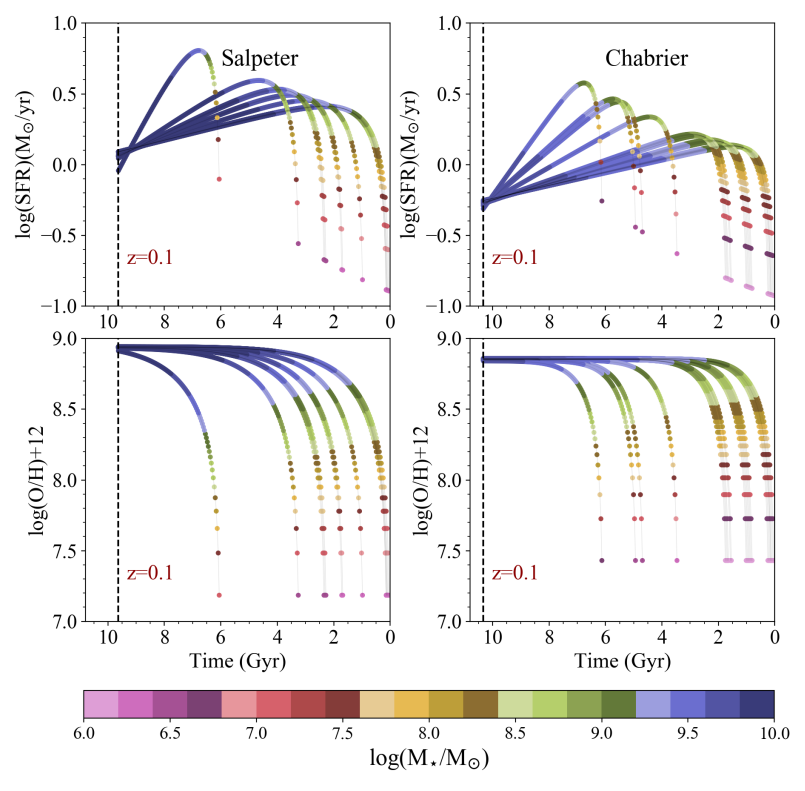

Fig. A.6. Evolution of star formation rate (upper panels) and oxygen abundance (lower panels) as a function of time for the galaxies with infall mass $M_{\text {inf }}=10^{10.5} M_{\odot}$ ) that lie on the MZR of Kewley \& Ellison (2008) and on the MSR as derived by Peng et al. (2010), computed assuming a Salpeter (1955) IMF (left panels) and a Chabrier (2003) IMF (right panels). The color-coding stands for the stellar mass.

In Fig. A.6 we compare the temporal evolution of the oxygen gas-phase abundances and of the SFR computed with two different IMFs. These quantities were computed for a set of model galaxies that locally lie on both the MZR and on the MSR and that are characterized by a constant infall mass, fixed at the value of $M_{\text {inf }}=10^{10.5} M_{\odot}$

As expected, the metallicity of the galaxies with a Chabrier (2003) IMF reach their saturation value at earlier times than with the Salpeter (1955) IMF. Stronger winds are required to avoid an excessive increase in their metallicity, with the consequence of a significant removal of the gas available for star formation. This implies that given the same infall mass, galaxies with a Chabrier (2003) IMF are forced to evolve at a lower star formation regime.

With a Chabrier (2003) IMF, the metallicity grows faster, but stellar mass grows at a slower pace than with a Salpeter (1955) IMF. In the models shown in Fig. A.6 the SFH is continuous, and because of a more efficient gas removal in the case of the Chabrier (2003) IMF, the only way to have comparable stellar masses at the present day is by means of a more extended SFH.

It is worth noting that in the case of Fig. A.6, the models with a Chabrier (2003) IMF will have lower stellar masses than those with a Salpeter IMF. However, the same considerations will hold also for galaxies with the same present-day stellar masses which, in the case of a Chabrier (2003) IMF, will need to have started forming stars at earlier epochs, and which will thus present on average older mass-weighted ages. With reference to the galaxies drawn in Fig. A.6 using the Salpeter (1955) IMF, the average values for the stellar mass, the temporal evolution $t_{n}$ computed at redshift $z=0.1$, and the associated massweighted age $\operatorname{Age}\left(t_{n}\right)$ are $\log \left(M_{\star} / M_{\odot}\right)=10.09, t_{n}=8.15 \mathrm{Gyr}$, and $\operatorname{Age}\left(t_{n}\right)=4.45 \mathrm{Gyr}$. On the other hand, with the Chabrier (2003) IMF, we have that $\log \left(M_{\star} / M_{\odot}\right)=9.71, t_{n}=8.90 \mathrm{Gyr}$, and $\operatorname{Age}\left(t_{n}\right)=5.11$ Gyr. On average, the model with the Chabrier (2003) IMF therefore predicts older objects with a smaller stellar mass content. 